J. Appl. Numer. Optim. 3 (2021), No. 3, pp. 435-456

Available online at http://jano.biemdas.com

https://doi.org/10.23952/jano.3.2021.3.01

\title{
THE FUN IS FINITE: DOUGLAS-RACHFORD AND SUDOKU PUZZLE - FINITE TERMINATION AND LOCAL LINEAR CONVERGENCE
}

\author{
ROBERT TOVEY ${ }^{1}$, JINGWEI LIANG ${ }^{2, *}$ \\ ${ }^{1}$ INRIA Paris, France \\ ${ }^{2}$ Institute of Natural Sciences and School of Mathematical Sciences, \\ Shanghai Jiao Tong University, Shanghai, China
}

\begin{abstract}
In recent years, the Douglas-Rachford splitting method has been shown to be effective at solving many non-convex optimization problems. In this paper, a local convergence analysis for nonconvex feasibility problems is presented and both finite termination and local linear convergence are obtained. For a generalization of the Sudoku puzzle, it is proved that the local linear rate of convergence of Douglas-Rachford is exactly $\frac{\sqrt{5}}{5}$ and independent of puzzle size. For the $s$-queens problem, it is proved that Douglas-Rachford converges after a finite number of iterations. Numerical results on solving Sudoku puzzles and $s$-queens puzzles are provided to support our theoretical findings.

Keywords. Douglas-Rachford; Feasibility problem; Finite termination; Local Linear Convergence; Sudoku puzzle.
\end{abstract}

\section{INTRODUCTION}

Given two non-empty sets $C$ and $S$ whose intersection is also non-empty, the feasibility problem aims to find a common point in the intersection $C \cap S$. In the literature, popular numerical schemes for solving feasibility problems were developed based on projections. Among them, the alternating projection is the fundamental one. The method of the alternating projection was first introduced by von Neumann for the case of two linear subspaces [1], then it was extended to closed convex sets by Bregman [2]. Relaxation is a standard approach to speed up the alternating projection. For related work, we refer to $[3,4]$ and the references therein.

Proximal splitting methods, such as Forward-Backward [5] splitting and Backward-Backward splitting [6], and Peaceman-Rachford/Douglas-Rachford splitting [7, 8], can also be applied to solve feasibility problems either directly or up to reformulation. Moreover, the equivalence between projection-based methods and proximal splitting methods can be established, such as the alternating projection is equivalent to Backward-Backward splitting while the relaxed alternating relaxed projection covers Peaceman-Rachford/Douglas-Rachford splitting as special cases [6].

Our focus in this paper is the Douglas-Rachford splitting method, which has shown to be effective for solving feasibility problems, particularly in the non-convex setting [9]. However,

${ }^{*}$ Corresponding author.

E-mail addresses: robert.tovey @inria.fr (R. Tovey), jingwei.liang @sjtu.edu.cn (J. Liang).

Received November 1, 2021; Accepted December 5, 2021.

(C)2021 Journal of Applied and Numerical Optimization 
the convergence property is rather less understood than its convex counterpart. One reason for this is that the Douglas-Rachford splitting method is not symmetric and non-descent, when compared to the (proximal) gradient descent whose non-convex case is much easier studied [10]. Research on non-convex Douglas-Rachford either focuses on specific cases or imposing stronger assumptions (e.g. smoothness) and proposes modifications to the original iteration. For instance, the authors [11] considered the Douglas-Rachford splitting for solving the feasibility problem of a line intersecting with a circle, and the conditions for convergence. In [12], the authors proposed a damped Douglas-Rachford splitting method for general non-convex optimization problems under the condition that one function has a Lipschitz continuous gradient.

The study of this paper is motivated by applying the Douglas-Rachford to solve Sudoku puzzle, for which three different convergence behaviors are observed

- Globally, the method converges sub-linearly.

- Locally, two regimes occur: finite termination and linear convergence.

Finite termination and local linear convergence were reported in the literatures [9, 13], however, the conditions in respective work either were designed for convex setting or cannot be satisfied by Sudoku puzzle. Therefore, a new analysis is needed for the Douglas-Rachford splitting which is the aim of this paper:

(1) Finite termination Under a non-degeneracy condition (see (4.1)), we show in Section 4 that one sequence generated by the Douglas-Rachford splitting has the finite termination property. All sequences terminate in a finite number of iterations if the problem satisfies certain assumptions (e.g. polyhedrality, see Assumptions (A.1)-(A.3)).

(2) Local linear convergence We also provide a precise characterization for the local linear convergence of the Douglas-Rachford splitting method. Particularly, for Sudoku puzzle, we prove that the linear rate of convergence of Douglas-Rachford splitting method is precisely $\frac{\sqrt{5}}{5}$. Moreover, such a rate is independent of the puzzle size. For the damped Douglas-Rachford splitting method, we also provide an exact estimation of the local linear rate, which depends on the damping coefficient.

Relation to prior work There are several existing work on the finite termination property of the standard Douglas-Rachford splitting method. In [13], the authors established the finite convergence of Douglas-Rachford in the presence of Slater's condition for solving the convex feasibility problems, where one set is an affine subspace, and the other is a polyhedron, or one set is an epigraph, and the other one is a hyperplane. The result was extended to general convex optimization problems in [14] under the notion of partial smoothness [15]. In [16], finite termination was proved for finding a point which is guaranteed to be in the interior of one set whose interior is assumed to be non-empty. The result of [13] was later extended to the non-convex case in [9], where one of the two sets can be finite.

For the local linear convergence, results can be found in, for instance, [17], where the linear convergence of the Douglas-Rachford splitting method was established under a regularity condition. Similar results can be found in $[18,19]$. Under a constraint qualification condition, the local linear convergence property of the damped Douglas-Rachford splitting method was discussed in [12].

https://en.wikipedia.org/wiki/Sudoku 
Paper organization The rest of the paper is organized as follows. Some preliminaries are collected in Section 2. Section 3 states our main assumptions on problem (3.1), and introduces the standard and damped Douglas-Rachford algorithms. The global convergence is also discussed in this section. Our main result on local convergence of Douglas-Rachford is presented in Section 4. In Section 5, we report numerical experiments on Sudoku puzzle and s-queens puzzle to support our theoretical findings. In Section 6, the last section, we give the concluding remarks.

\section{PRELIMINARIES}

Throughout the paper, $\mathbb{N}$ is the set of nonnegative integers, and $\mathbb{R}^{n}$ is a finite $n$-dimensional real Euclidean space equipped with scalar product $\langle\cdot, \cdot\rangle$ and norm $\|\cdot\|$. Id denotes the identity operator on $\mathbb{R}^{n}$. For a matrix $M \in \mathbb{R}^{n \times n}$, we denote $\rho(M)$ its spectral radius.

Projection and reflection Below we collect necessary concepts related to sets.

Definition 2.1 (Distance and indicator function). Let $C \subset \mathbb{R}^{n}$ be non-empty and $x \in \mathbb{R}^{n}$. The distance function of $x$ to $C$ is defined by

$$
\operatorname{dist}(x, C) \stackrel{\text { def }}{=} \inf _{y \in C}\|x-y\| .
$$

The indicator function of $C$ is defined by $\iota_{C}(x)=\left\{\begin{array}{r}0: x \in C, \\ +\infty: x \notin C\end{array}\right.$

Definition 2.2 (Projection \& reflection). Let $C \subset \mathbb{R}^{n}$ be non-empty and $x \in \mathbb{R}^{n}$. The projection of $x$ onto $C$, denoted by $\mathscr{P}_{C}(x)$, is a set defined by

$$
\mathscr{P}_{C}(x) \stackrel{\text { def }}{=}\{y \in C:\|x-y\|=\operatorname{dist}(x, C)\} .
$$

The mapping $\mathscr{P}_{C}: \mathbb{R}^{n} \rightrightarrows C$ is called the projection operator. The relaxed projection $\mathscr{P}_{C}^{\lambda}$ is defined via

$$
\mathscr{P}_{C}^{\lambda}(x)=\lambda \mathscr{P}_{C}(x)+(1-\lambda) x
$$

where $\lambda \in] 0,2]$ is the relaxation parameter. When $\lambda=2$, the corresponding mapping is called reflection and denoted by $\mathscr{R}_{C}(x)=2 \mathscr{P}_{C}(x)-x$.

Definition 2.3 (Prox-regularity). A non-empty closed set $C \subset \mathbb{R}^{n}$ is prox-regular at $x \in C$ for $v$ if $x=\mathscr{P}_{C}(x+v)$. If $\mathscr{P}_{C}$ is single-valued in an open neighborhood of $x \in C$, then $C$ is said to be prox-regular at $x$.

Definition 2.4 (Normal vector). Given $C \subset \mathbb{R}^{n}$ and $x \in C$, the proximal normal cone $\mathscr{N}_{C}^{p}(x)$ of $C$ at $x$ is defined by

$$
\mathscr{N}_{C}^{p}(x)=\operatorname{cone}\left(\mathscr{P}_{C}^{-1}(x)-x\right) .
$$

The limiting normal cone $\mathscr{N}_{C}(x)$ is defined as any vector that can be written as the limit of proximal normals: $v \in \mathscr{N}_{C}(x)$ if and only if there exists sequences $\left\{x_{k}\right\}_{k \in \mathbb{N}} \in C$ and $\left\{v_{k}\right\}_{k \in \mathbb{N}}$ in $\mathscr{N}_{C}\left(x_{k}\right)$ such that $x_{k} \rightarrow x$ and $v_{k} \rightarrow v$.

Let $C_{1}, C_{2} \subset \mathbb{R}^{n}$ be two sets with non-empty intersection. The feasibility problem of $C_{1}, C_{2}$ is to find a common point in the intersection, i.e.

$$
\text { find } \quad x \in \mathbb{R}^{n} \quad \text { s.t. } \quad x \in C_{1} \bigcap C_{2} \text {. }
$$


A fundamental algorithm to solve the problem is the alternating projection method which, as indicated by the name, represents the procedure: from a given point $x_{0}$, apply projection onto each set alternatively

$$
x_{k+1}=\mathscr{P}_{C_{2}} \mathscr{P}_{C_{1}}\left(x_{k}\right) .
$$

One can also consider relaxations for each projection operator and the whole iteration, which results in the following iteration

$$
x_{k+1}=x_{k}+\lambda\left(\mathscr{P}_{C_{2}}^{\lambda_{2}} \mathscr{P}_{C_{1}}^{\lambda_{1}}\left(x_{k}\right)-x_{k}\right),
$$

where $\lambda, \lambda_{1}$, and $\lambda_{2}$ are relaxation parameters. The iteration becomes Peaceman-Rachford splitting (alternating reflection) for $\left(\lambda, \lambda_{1}, \lambda_{2}\right)=(1,2,2)$ and Douglas-Rachford splitting for $\left(\lambda, \lambda_{1}, \lambda_{2}\right)=(1 / 2,2,2)$. We refer to [20] for a survey on the alternating projection method.

Convergent matrices To discuss the local linear convergence, we need the following preliminary results on convergent matrices which are taken from [21].

Definition 2.5 (Convergent matrices). A matrix $M \in \mathbb{R}^{n \times n}$ is said to be convergent to $\mathrm{M}^{\infty} \in$ $\mathbb{R}^{n \times n}$ if and only if $\lim _{k \rightarrow+\infty}\left\|M^{k}-\mathrm{M}^{\infty}\right\|=0$. $M$ is said to be linearly convergent if there exists $\eta \in\left[0,1\left[\right.\right.$ and $K \in \mathbb{N}$ such that, for all $k \geq K$, there holds $\left\|M^{k}-\mathrm{M}^{\infty}\right\|=O\left(\eta^{k}\right)$. If $M$ does not converge at any rate $\eta^{\prime} \in[0, \eta[$, then $\eta$ is called the optimum convergence rate.

Definition 2.6 (Semi-simple eigenvalue). For $M \in \mathbb{R}^{n \times n}$, an eigenvalue $\eta$ is said to be semisimple if and only if $\operatorname{rank}(M-\eta \mathrm{Id})=\operatorname{rank}\left((M-\eta \mathrm{Id})^{2}\right)$.

Theorem 2.1 (Limits of powers). For $M \in \mathbb{R}^{n \times n}$, the power of $M$ converges to $\mathrm{M}^{\infty}$ if and only if $\rho(M)<1$ or $\rho(M)=1$ with 1 being the only eigenvalue on the complex unit circle and semi-simple.

Whenever $M$ is convergent, it converges linearly to $\mathrm{M}^{\infty}$, and we have the following lemma.

Lemma 2.1 (Convergence rate). If $M \in \mathbb{R}^{n \times n}$ is convergent to some $\mathrm{M}^{\infty} \in \mathbb{R}^{n \times n}$, then

(i) for any $k \in \mathbb{N}$,

$$
M^{k}-\mathrm{M}^{\infty}=\left(M-\mathrm{M}^{\infty}\right)^{k} \quad \text { and } \quad\left\|M^{k}-\mathrm{M}^{\infty}\right\| \leq\left\|M-\mathrm{M}^{\infty}\right\|^{k} .
$$

The equality holds only when $M$ is normal.

(ii) We have that $\rho\left(M-\mathrm{M}^{\infty}\right)<1$, and $M$ is linearly convergent for any $\left.\eta \in\right] \rho\left(M-\mathrm{M}^{\infty}\right), 1[$.

(iii) $\rho\left(M-\mathrm{M}^{\infty}\right)$ is the optimal convergence rate if one of the following holds

(a) $M$ is normal;

(b) All the eigenvalues $\eta \in \Theta_{M}$ such that $|\eta|=\rho\left(M-\mathrm{M}^{\infty}\right)$ are semi-simple.

Proof. See Theorems 2.12, 2.13, 2.15 and 2.16 of [22].

Angles between subspaces To precisely characterize the local linear convergence rate, we need the following concepts regarding the angles between subspaces. Let $T_{1}$ and $T_{2}$ be two linear subspaces with dimension $p \stackrel{\text { def }}{=} \operatorname{dim}\left(T_{1}\right)$ and $q \stackrel{\text { def }}{=} \operatorname{dim}\left(T_{2}\right)$. Without loss of generality, we suppose that $1 \leq p \leq q \leq n-1$. 
Definition 2.7 (Principal angles). The principal angles $\theta_{k} \in\left[0, \frac{\pi}{2}\right], k=1, \ldots, p$ between linear subspaces $T_{1}$ and $T_{2}$ are defined by, with $u_{0}=v_{0} \stackrel{\text { def }}{=} 0$ and inductively

$$
\begin{aligned}
\cos \left(\theta_{k}\right) \stackrel{\text { def }}{=}\left\langle u_{k}, v_{k}\right\rangle=\max \left\{\langle u, v\rangle \text { s.t. } u \in T_{1}, v \in T_{2},\|u\|=1,\|v\|=1,\right. \\
\left.\left\langle u, u_{i}\right\rangle=\left\langle v, v_{i}\right\rangle=0, i=0, \cdots, k-1\right\} .
\end{aligned}
$$

The principal angles $\theta_{k}$ are unique with $0 \leq \theta_{1} \leq \theta_{2} \leq \cdots \leq \theta_{p} \leq \pi / 2$.

Definition 2.8 (Friedrichs angle). The Friedrichs angle $\theta_{F} \in\left[0, \frac{\pi}{2}\right]$ between $T_{1}$ and $T_{2}$ is

$$
\begin{aligned}
\cos \left(\theta_{F}\left(T_{1}, T_{2}\right)\right) \stackrel{\text { def }}{=} \max \langle u, v\rangle \text { s.t. } u & \in T_{1} \cap\left(T_{1} \cap T_{2}\right)^{\perp},\|u\|=1, \\
v & \in T_{2} \cap\left(T_{1} \cap T_{2}\right)^{\perp},\|v\|=1 .
\end{aligned}
$$

The following lemma shows the relation between the Friedrichs and principal angles.

Lemma 2.2 ([22, Proposition 3.3]). We have $\theta_{F}\left(T_{1}, T_{2}\right)=\theta_{d+1}>0$, where $d \stackrel{\text { def }}{=} \operatorname{dim}\left(T_{1} \cap T_{2}\right)$.

\section{The Problem And Algorithms}

The formal statement of the feasibility problem is written below

$$
\text { find } \quad x \in \mathbb{R}^{n} \quad \text { s.t. } \quad x \in C \bigcap S,
$$

where the following assumptions are imposed

(A.1) $C \subset \mathbb{R}^{n}$ is a closed set;

(A.2) $S \subset \mathbb{R}^{n}$ is an affine subspace;

(A.3) $C \bigcap S \neq \emptyset$, i.e. the intersection is non-empty.

Note that problem (3.1) is not necessarily convex as we suppose that $C$ is only non-empty and closed. The examples of (3.1) are provided in Section 4, including the Sudoku puzzle and $s$-queens puzzle.

3.1. Douglas-Rachford splitting method. The development of the Douglas-Rachford (DR) splitting method [8] dates back to 1950s for solving numerical PDEs. In recently years, the method has also been shown to be effective for non-convex feasibility problems; see, e.g., $[23,24]$ and the references therein. Details of the method for solving (3.1) is described in Algorithm 1.

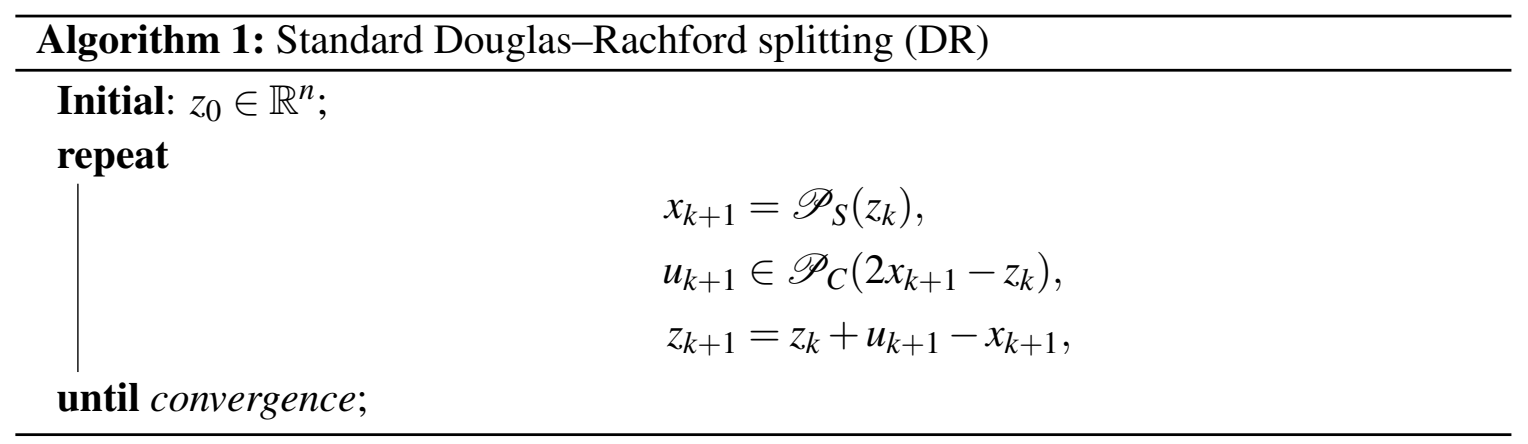

The above iteration can be written as the fixed-point iteration of variable $z_{k}$. Denote the fixed-point operator

$$
\mathscr{F}_{\mathrm{DR}} \stackrel{\text { def }}{=} \frac{1}{2}\left(\left(2 \mathscr{P}_{C}-\mathrm{Id}\right)\left(2 \mathscr{P}_{S}-\mathrm{Id}\right)+\mathrm{Id}\right)
$$

Then, $z_{k+1}=\mathscr{F}_{\mathrm{DR}}\left(z_{k}\right)$. The other two variables $u_{k}, x_{k}$ are called the shadow sequences [25]. 
Determining the convergence properties of the Douglas-Rachford splitting for the non-convex setting is a challenging problem since the non-descent property of the method makes it much harder to obtain convergence results than the descent-type methods, which include (proximal) gradient descent [10].

Moreover, since the method has three different sequences $u_{k}, x_{k}$, and $z_{k}$, various different convergence behaviors may occur. We refer to [9] for more detailed discussions. In Example 3.1, we demonstrate a case of a circle intersecting with a line where:

- the shadow sequences $\left\{u_{k}\right\}_{k \in \mathbb{N}},\left\{x_{k}\right\}_{k \in \mathbb{N}}$ converge to $u^{\star}$ and $x^{\star}$, respectively. But $u^{\star} \neq$ $x^{\star}$

- the fixed-point sequence $\left\{z_{k}\right\}_{k \in \mathbb{N}}$ diverges.

As our main interest in this paper is to study the local behavior, for the rest of the paper, we suppose that the standard DR is globally convergent:

(A.4) the standard Douglas-Rachford splitting method for solving (3.1) is globally convergent.

Consequently, one has

$$
z_{k} \rightarrow z^{\star} \in \operatorname{Fix}(\mathscr{F} \text { DR }) \stackrel{\text { def }}{=}\left\{z \in \mathbb{R}^{n}: z=\mathscr{F}_{\mathrm{DR}}(z)\right\} \quad \text { and } \quad u_{k}, x_{k} \rightarrow x^{\star} \in \mathscr{P}_{S}\left(z^{\star}\right) .
$$

To avoid assumption (A.4), people either turn to specific cases [26] or imposing stronger assumptions, such as smoothness [27]. Modifications to the original Douglas-Rachford splitting method were also considered in the literature. Below we describe a damped version of the Douglas-Rachford splitting proposed in [12].

Solving feasibility problem (3.1) is equivalent to the following constrained smooth optimization

$$
\min _{x \in \mathbb{R}^{n}} \frac{1}{2} \operatorname{dist}^{2}(x, S) \quad \text { s.t. } \quad x \in C .
$$

In (3.2), the update of $x_{k+1}$ is equivalent to solving $\min _{x \in \mathbb{R}^{n}} l_{S}(x)+\frac{1}{2 \gamma}\left\|x-z_{k}\right\|^{2}$. Replacing the indicator function with the distance function,

$$
\min _{x \in \mathbb{R}^{n}} \frac{1}{2} \operatorname{dist}^{2}(x, S)+\frac{1}{2 \gamma}\left\|x-z_{k}\right\|^{2},
$$

we then obtain

$$
x_{k+1}=\frac{1}{1+\gamma}\left(z_{k}+\gamma \mathscr{P}_{S}\left(z_{k}\right)\right)=z_{k}+\frac{\gamma}{1+\gamma}\left(\mathscr{P}_{S}\left(z_{k}\right)-z_{k}\right)=\mathscr{P}_{S}^{\frac{\gamma}{1+\gamma}}\left(z_{k}\right) .
$$

As a result, we obtain the algorithm proposed in [12].

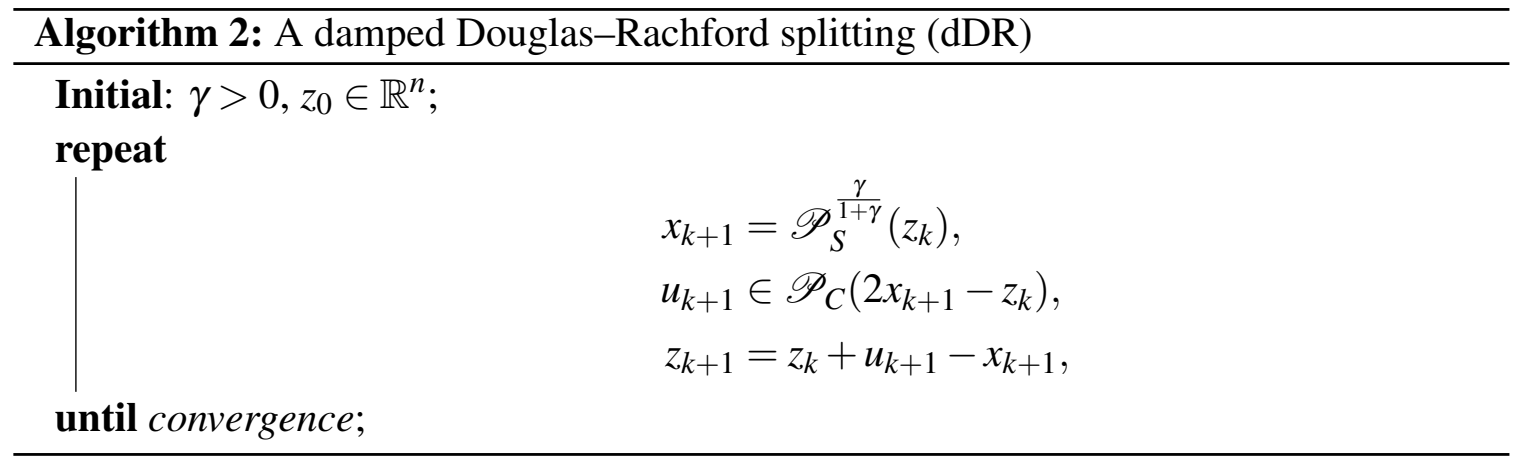


We refer to the original work [12] for a more detailed discussion of Algorithm 2. When $\gamma=+\infty$, Algorithm 2 recovers standard Douglas-Rachford splitting method (3.2). The fixedpoint operator of $\mathrm{dDR}$ reads

$$
\mathscr{F}_{\mathrm{dDR}} \stackrel{\text { def }}{=} \frac{1}{2}\left(\left(2 \mathscr{P}_{C}-\mathrm{Id}\right)\left(2 \mathscr{P}_{S}^{\frac{\gamma}{1+\gamma}}-\mathrm{Id}\right)+\mathrm{Id}\right) .
$$

We have the following convergence result of the dDR from [12].

Lemma 3.1 (Global convergence of dDR [12, Theorem 5]). For non-convex feasibility problem (3.1), suppose that Assumptions (A.1)-(A.3) hold and moreover $C$ is compact. Choose $\gamma \in] 0, \sqrt{3 / 2}-1\left[\right.$ for Douglas-Rachford splitting method (3.4). Then $\left\{u_{k}, x_{k}, z_{k}\right\}_{k \in \mathbb{N}}$ is bounded, and given any cluster point $\left(u^{\star}, x^{\star}, z^{\star}\right)$ of the sequence, there holds $\left\|z_{k}-z_{k-1}\right\| \rightarrow 0, u^{\star}=x^{\star}$, and $x^{\star}$ is a stationary point of problem (3.3).

In the example below, we demonstrate a case, where DR fails to solve the problem while dDR succeeds.

Example 3.1 (A circle intersects with a line). Let $C=\left\{x \in \mathbb{R}^{2}:\|x\|=1\right\}$ be the unit circle, and let $S=\left\{x \in \mathbb{R}^{2}:\left\langle x,\left(\begin{array}{l}1 \\ 2\end{array}\right)\right\rangle=\sqrt{2}\right\}$ be a line that intersects with $C$ at two different points. For both methods, same initial point $z_{0}=(-10,-8)$ is chosen. For damped DR, we set $\gamma=\frac{1}{5}$. In Figure 1, we observe:

- for the standard DR (left): $z_{k}$ is not convergent, $u_{k}$ and $x_{k}$ converge to two different points, and the method fails to find a feasible point.

- for the damped DR (right): all three sequences converge to the same feasible point.

We refer to [11] for a detailed discussion of the convergence properties of the standard DR for solving this feasibility problem.

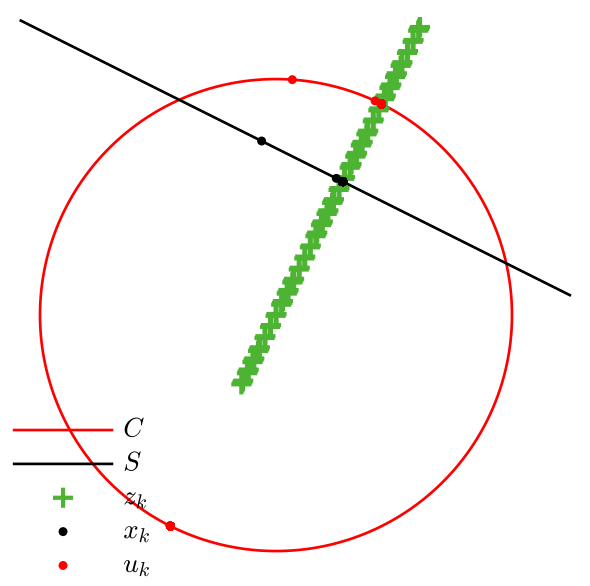

(A) Standard Douglas-Rachford

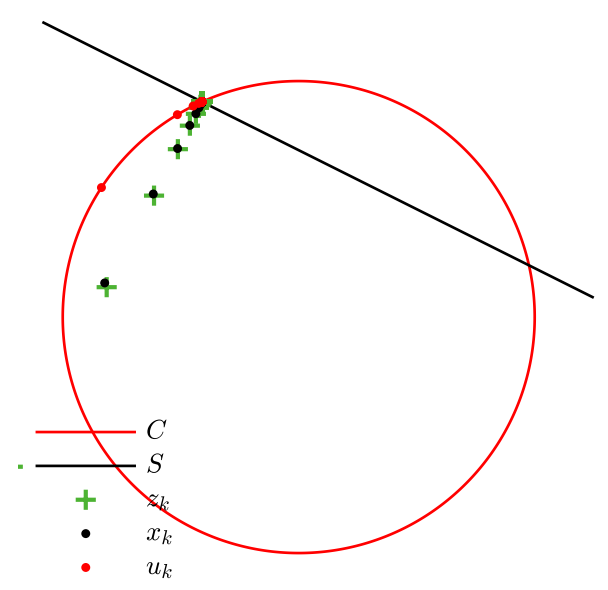

(B) Damped Douglas-Rachford

FigurE 1. Convergence behavior of the standard and damped DouglasRachford for solving the problem of a line intersecting with a circle.

Though dDR solves the problem, as remarked in the original paper [12], it may also converges to some stationary point of (3.3), which is not a solution. In fact, as we shall see in the numerical 
experiments, for the Sudoku puzzle and the $s$-queens puzzle, dDR with $\left.\gamma=\frac{1}{5} \in\right] 0, \sqrt{3 / 2}-1[$ fails all tests while sDR achieves very good performance; see Table 1.

3.2. Problems with more than two sets. Up to now, we have been dealing with feasibility problem of two sets, while in various scenarios we need to deal with the case of finding common points of more than two sets. In what follows, we briefly show that, by a product space trick, we can reformulate the problem into the form of (3.1).

Let $m \geq 2$ be an integer, and let $C_{i}$ be a non-empty closed set for each $i \in\{1, \ldots, m\}$. Consider the following feasibility problem

$$
\text { find } \quad x \in \mathbb{R}^{n} \quad \text { s.t. } \quad x \in \bigcap_{i=1}^{m} C_{i} .
$$

Let $\mathcal{H}=\underbrace{\mathbb{R}^{n} \times \cdots \times \mathbb{R}^{n}}_{m \text { times }}$ be the product space endowed with the scalar inner-product and norm

$$
\forall \boldsymbol{x}, \boldsymbol{y} \in \mathcal{H},\langle\boldsymbol{x}, \boldsymbol{y}\rangle=\sum_{i=1}^{m}\left\langle x_{i}, y_{i}\right\rangle,\|\boldsymbol{x}\|=\left(\sum_{i=1}^{m}\left\|x_{i}\right\|^{2}\right)^{1 / 2} .
$$

Let $\mathcal{C} \stackrel{\text { def }}{=} C_{1} \times \cdots \times C_{m}$. Then $\mathcal{C} \subset \mathcal{H}$. Denote the subspace $\mathcal{S} \stackrel{\text { def }}{=}\left\{\boldsymbol{x}=\left(x_{i}\right)_{i} \in \mathcal{H}: x_{1}=\cdots=x_{m}\right\}$. The feasibility problem (3.5) can be reformulated into the following form

$$
\text { find } \boldsymbol{x} \in \mathcal{H} \quad \text { s.t. } \quad \boldsymbol{x} \in \mathcal{C} \cap \mathcal{S} \text {. }
$$

The projection operator of $\mathcal{C}$ is component-wise for each set $C_{i}, i=1, \ldots, m$,

$$
\mathscr{P}_{\mathcal{C}} \boldsymbol{x}=\left(\mathscr{P}_{C_{1}} x_{1}, \cdots, \mathscr{P}_{C_{m}} x_{m}\right) .
$$

Defining $\mathcal{K}: \mathbb{R}^{n} \rightarrow \mathcal{S}, x \mapsto(x, \cdots, x)$, we have $\mathscr{P}_{\mathcal{S}}(\boldsymbol{x})=\mathcal{K}\left(\frac{1}{m} \sum_{i=1}^{m} x_{i}\right)$.

Adapting the standard Douglas-Rachford to the case of (3.5), we obtain

$$
\begin{aligned}
& x_{k+1}=\frac{1}{m} \sum_{i=1}^{m} z_{i, k}, \\
& \text { For } i=1, \ldots, m: \\
& \qquad \begin{array}{l}
u_{i, k+1} \in \mathscr{P}_{C_{i}}\left(2 x_{k+1}-z_{i, k}\right), \\
z_{i, k+1}=z_{i, k}+u_{i, k+1}-x_{k+1} .
\end{array}
\end{aligned}
$$

Note that for the standard DR, there is no need to store $\boldsymbol{x}$ and simply $x_{k+1}=\frac{1}{m} \sum_{i=1}^{m} z_{i, k}$ is sufficient. Correspondingly, we also have the following iteration for the damped DouglasRachford splitting method:

$$
\begin{aligned}
& \text { For } i=1, \ldots, m: \\
& \qquad \begin{array}{l}
x_{i, k+1}=\frac{1}{1+\gamma}\left(z_{i, k}+\gamma \frac{1}{m} \sum_{j=1}^{m} z_{j, k}\right), \\
u_{i, k+1} \in \mathscr{P}_{C_{i}}\left(2 x_{i, k+1}-z_{i, k}\right), \\
z_{i, k+1}=z_{i, k}+u_{i, k+1}-x_{i, k+1} .
\end{array}
\end{aligned}
$$

\section{Local Convergence of the Douglas-Rachford SplitTing}

In this section, we present our main result, the local convergence of Douglas-Rachford splitting. We first present the result in a general setting, and then specialize to the case of Sudoku and $s$-queens puzzles.

Non-degeneracy condition To deliver the result, a non-degeneracy condition is needed for set C. Assume Assumption (A.4) holds for standard DR and that dDR is ran under the condition 
of Lemma 3.1. Then, at convergence for both methods, we have $z_{k} \rightarrow z^{\star}$ and $u_{k}, x_{k} \rightarrow x^{\star}$. We assume that $C$ is prox-regular at $x^{\star}$ for $x^{\star}-z^{\star}$ and the following condition holds

$$
x^{\star}-z^{\star} \in \operatorname{int}\left(\mathscr{N}_{C}\left(x^{\star}\right)\right)
$$

where int $(\cdot)$ stands for the interior of the set.

Remark 4.1. The non-degeneracy condition (4.1) requires $\mathscr{N}_{C}\left(x^{\star}\right)$ has a non-empty interior, which means that $x^{\star}$ is a vertex of the set $C$. A graphical illustration of the non-degeneracy condition (4.1) is provided in Figure 2 below.

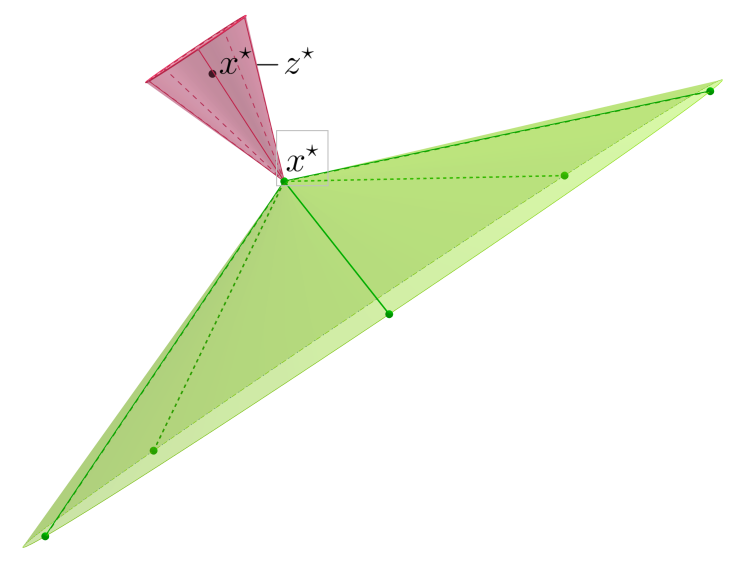

FIGURE 2. Normal cone (red) at a point $x^{*}$ in the polytope $C$ (green).

4.1. Local convergence of Douglas-Rachford splitting. We start with the standard DouglasRachford splitting and then the damped iteration. Relation with some existing work in the literature is also discussed.

4.1.1. The standard Douglas-Rachford splitting. For the standard Douglas-Rachford splitting method, for what follows, we impose the global convergence as an assumption, i.e. (A.4) holds.

Theorem 4.1 (Finite termination of DR). For feasibility problem (3.1) and Douglas-Rachford iteration (3.2), suppose that Assumptions (A.1)-(A.4) hold. Then $\left\{u_{k}, x_{k}, z_{k}\right\}_{k \in \mathbb{N}}$ converges to $\left(x^{\star}, x^{\star}, z^{\star}\right)$ with $z^{\star} \in \operatorname{Fix}(\mathscr{F} \mathrm{DR})$ being a fixed point and $x^{\star}=\mathscr{P}_{S}\left(z^{\star}\right)$. If, moreover, nondegeneracy condition (4.1) holds, then $\left\{u_{k}, x_{k}, z_{k}\right\}_{k \in \mathbb{N}}$ converges to $\left(x^{\star}, x^{\star}, z^{\star}\right)$ finitely.

Remark 4.2. It is worth noting that Theorem 4.1 also holds true for the convex setting. In [13], the authors studied the DR for solving convex affine-polyhedral feasibility problems, and imposed the following condition for finite convergence

$$
S \bigcap \operatorname{int}(C) \neq \emptyset
$$

which does not hold for the non-convex case as the interior of $C$ in (3.1) may be empty (see also Section 4.2 the puzzles for which (4.2) fails). In [9], when the non-convex set is finite, the finite termination was proved given that the other set is an affine subspace or a half-space. In comparison, our result here does not need the set to be finite and provides an extension to that of [13], as we characterize the situation where finite convergence happens but (4.2) fails. 
Proof. The imposed global convergence of (3.2) means

$$
z_{k} \rightarrow z^{\star} \in \operatorname{Fix}\left(\mathscr{F}_{\mathrm{DR}}\right) \quad \text { and } \quad u_{k}, x_{k} \rightarrow x^{\star}=\mathscr{P}_{S}\left(z^{\star}\right) .
$$

The prox-regularity of $C$ at $x^{\star}$ for $x^{\star}-z^{\star}$ and the non-degeneracy condition (4.1) imply that there exists an open set $\mathscr{B}$ such that

$$
2 x^{\star}-z^{\star} \in \mathscr{B} \subset \mathscr{N}_{C}\left(x^{\star}\right)+x^{\star} \quad \text { and } \quad \mathscr{P}_{C}(\mathscr{B})=\left\{x^{\star}\right\} .
$$

By the definition of convergence, one concludes that there exists $K \in \mathbb{N}$ such that $2 x_{k+1}-z_{k+1} \in$ $\mathscr{B}$ for all $k \geq K$. Consequently, by the update of $u_{k+1}$ in (3.4), one has

$$
u_{k+1}=\mathscr{P}_{C}\left(2 x_{k+1}-z_{k}\right)=x^{\star},
$$

which is the finite convergence of $u_{k+1}$. For the update of $x_{k}$ in (3.2), this time we have directly

$$
x_{k+1}-x^{\star}=\mathscr{P}_{S}\left(z_{k}-z^{\star}\right) \text {. }
$$

For $z_{k+1}$, let $K>0$ be such that $u_{k}=x^{\star}$ for all $k \geq K$. It follows that

$$
\begin{aligned}
z_{k+1}-z^{\star}=\left(z_{k}-z^{\star}\right)+\left(u_{k+1}-x^{\star}\right)-\left(x_{k+1}-x^{\star}\right) & =\left(z_{k}-z^{\star}\right)-\left(x_{k+1}-x^{\star}\right) \\
& =\left(\operatorname{Id}-\mathscr{P}_{S}\right)\left(z_{k}-z^{\star}\right) \\
& =\left(\mathrm{Id}-\mathscr{P}_{S}\right)^{k+1-K}\left(z_{K}-z^{\star}\right) .
\end{aligned}
$$

Since $z_{k} \rightarrow z^{\star}$ and $\left(\mathrm{Id}-\mathscr{P}_{S}\right)^{k+1-K}=\mathrm{Id}-\mathscr{P}_{S}$, we have

$$
0=\lim _{k \rightarrow+\infty} z_{k+1}-z^{\star}=\lim _{k \rightarrow+\infty}\left(\operatorname{Id}-\mathscr{P}_{S}\right)^{k+1-K}\left(z_{K}-z^{\star}\right)=\left(\operatorname{Id}-\mathscr{P}_{S}\right)\left(z_{K}-z^{\star}\right)=z_{k+1}-z^{\star},
$$

which means $z_{k}=z^{\star}$ for all $k>K$, the finite termination of $z_{k}$. The finite convergence of $x_{k}$ follows naturally that of $z_{k}$, and we conclude the proof.

Different order of update In (3.2), the order of the projection operators can be switched which results in the following iteration

$$
\begin{aligned}
& x_{k+1} \in \mathscr{P}_{C}\left(z_{k}\right), \\
& u_{k+1}=\mathscr{P}_{S}\left(2 x_{k+1}-z_{k}\right), \\
& z_{k+1}=z_{k}+u_{k+1}-x_{k+1} .
\end{aligned}
$$

The corollary below shows that the finite termination holds for (4.3).

Corollary 4.1. For feasibility problem (3.1) and Douglas-Rachford iteration (4.3), suppose that Assumptions (A.1)-(A.4) hold. Then $\left\{u_{k}, x_{k}, z_{k}\right\}_{k \in \mathbb{N}}$ converges to $\left(x^{\star}, x^{\star}, z^{\star}\right)$ with $z^{\star} \in \operatorname{Fix}(\mathscr{F} \mathrm{DR})$ being a fixed point and $x^{\star} \in \mathscr{P}_{C}\left(z^{\star}\right)$. If, moreover, $C$ is prox-regular at $x^{\star}$ for $z^{\star}-x^{\star}$ and the following non-degeneracy condition holds,

$$
-\left(x^{\star}-z^{\star}\right) \in \operatorname{int}\left(\mathscr{N}_{C}\left(x^{\star}\right)\right)
$$

then $\left\{u_{k}, x_{k}, z_{k}\right\}_{k \in \mathbb{N}}$ converges to $\left(x^{\star}, x^{\star}, z^{\star}\right)$ in a finite number of iterations. 
Proof. Following the argument of the proof of Theorem 4.1, we can easily derive the finite termination of $x_{k}$ under new non-degeneracy condition (4.4). In turn, for $k$ large enough, we have for $u_{k+1}$ that

$$
\begin{aligned}
u_{k+1}-x^{\star} & =\mathscr{P}_{S}\left(2 x_{k+1}-z_{k}\right)-\mathscr{P}_{S}\left(2 x^{\star}-z^{\star}\right) \\
& =2 \mathscr{P}_{S}\left(x_{k+1}-x^{\star}\right)-\mathscr{P}_{S}\left(z_{k}-z^{\star}\right) \\
& =-\mathscr{P}_{S}\left(z_{k}-z^{\star}\right) .
\end{aligned}
$$

As a result for $z_{k}$,

$$
\begin{aligned}
z_{k+1}-z^{\star}=\left(z_{k}-z^{\star}\right)+\left(u_{k+1}-x^{\star}\right)-\left(x_{k+1}-x^{\star}\right) & =\left(z_{k}-z^{\star}\right)+\left(u_{k+1}-x^{\star}\right) \\
& =\left(\operatorname{Id}-\mathscr{P}_{S}\right)\left(z_{k}-z^{\star}\right),
\end{aligned}
$$

which is the same as the last part of proof of Theorem 4.1. Hence we conclude the proof immediately.

4.1.2. The damped Douglas-Rachford splitting. We now turn to the local convergence analysis of damped Douglas-Rachford splitting (3.4), for which we have the following result.

Theorem 4.2 (Local convergence of dDR). For feasibility problem (3.1) and damped DouglasRachford iteration (3.4), suppose that Assumptions (A.1)-(A.3) hold, and (3.4) is ran under the conditions of Theorem 3.1. Then $\left(u_{k}, x_{k}, z_{k}\right) \rightarrow\left(x^{\star}, x^{\star}, z^{\star}\right)$ with $z^{\star} \in \operatorname{Fix}(\mathscr{F} \mathrm{dDR})$ being a fixed point and $x^{\star}$ a stationary point of (3.3). If, moreover, condition (4.1) holds, then

(i) $u_{k}$ converges in finite number of iterations. $u_{k}=x^{\star}$.

(ii) Let $\eta=\frac{\gamma}{1+\gamma}$, it holds $\left\|z_{k}-z^{\star}\right\|=O\left(\eta^{k}\right)$.

Proof. The finite convergence of $u_{k}$ follows the argument of the proof of Theorem 4.1. For the update of $x_{k}$ in (3.4), since $S$ is an affine subspace, $\mathscr{P}_{S}$ is linear

$$
\begin{aligned}
x_{k+1}-x^{\star} & =\frac{1}{1+\gamma}\left(z_{k}+\gamma \mathscr{P}_{S}\left(z_{k}\right)\right)-x^{\star} \\
& =\frac{1}{1+\gamma}\left(z_{k}+\gamma \mathscr{P}_{S}\left(z_{k}\right)\right)-\frac{1}{1+\gamma}\left(x^{\star}+\gamma \mathscr{P}_{S}\left(z^{\star}\right)\right) \\
& =\frac{1}{1+\gamma}\left(z_{k}-x^{\star}\right)+\frac{\gamma}{1+\gamma} \mathscr{P}_{S}\left(z_{k}-z^{\star}\right) .
\end{aligned}
$$

Now, for $z_{k+1}$, let $K>0$ be such that $u_{k}=x^{\star}$ for all $k \geq K$. It follows that

$$
\begin{aligned}
z_{k+1}-z^{\star} & =\left(z_{k}-z^{\star}\right)+\left(u_{k+1}-u^{\star}\right)-\left(x_{k+1}-x^{\star}\right) \\
& =\left(z_{k}-z^{\star}\right)-\left(x_{k+1}-x^{\star}\right) \\
& =\left(z_{k}-z^{\star}\right)-\frac{1}{1+\gamma}\left(z_{k}-x^{\star}\right)-\frac{\gamma}{1+\gamma} \mathscr{P}_{S}\left(z_{k}-z^{\star}\right) \\
& =\frac{\gamma}{1+\gamma}\left(\mathrm{Id}-\mathscr{P}_{S}\right)\left(z_{k}-z^{\star}\right) .
\end{aligned}
$$

Note that the spectral radius of the matrix appears above is

$$
\rho\left(\frac{\gamma}{1+\gamma}\left(\mathrm{Id}-\mathscr{P}_{S}\right)\right)=\frac{\gamma}{1+\gamma},
$$

which is combined with the fact the matrix is symmetric and normal, we conclude from Lemma 2.1 that $\frac{\gamma}{1+\gamma}$ is the local linear convergence rate of $\left\|z_{k}-z^{\star}\right\|$. 
Remark 4.3. In [12], the authors also discussed the local linear convergence of the damped DR under the following constraint qualification condition

$$
\mathscr{N}_{S}\left(\mathscr{P}_{S}\left(x^{\star}\right)\right) \cap-\mathscr{N}_{C}\left(x^{\star}\right)=0 .
$$

As shown in [12, Proposition 2], such a condition allows to show $x^{\star} \in C \cap S$ and $z^{\star}=x^{\star}$; See Example 3.1 which satisfies the above condition. The update of $x_{k+1}$ in (3.4) yields

$$
\begin{aligned}
\frac{1+\gamma}{\gamma}\left(z^{\star}-x^{\star}\right)=z^{\star}-\mathscr{P}_{S}\left(z^{\star}\right) & \in \mathscr{N}_{S}\left(\mathscr{P}_{S}\left(x^{\star}\right)\right), \\
z^{\star}-x^{\star} & \in-\mathscr{N}_{C}\left(x^{\star}\right) .
\end{aligned}
$$

This implies that only the fixed-points $z^{\star}$ such that $z^{\star}=x^{\star}$ satisfy qualification condition (4.5). In comparison, our non-degeneracy condition is more general than (4.5) in the sense that we only focus on $\mathscr{N}_{C}\left(x^{\star}\right)$, and does not need the intersection of $\mathscr{N}_{S}\left(\mathscr{P}_{S}\left(x^{\star}\right)\right) \cap-\mathscr{N}_{C}\left(x^{\star}\right)$ to be 0 . Our result holds for all fixed-points of $\operatorname{Fix}\left(\mathscr{F}_{\mathrm{dDR}}\right)$.

Remark 4.4. If $S$, instead of being an affine subspace, has locally smooth curvature around $x^{\star}$, then, according to the result of [14], one can show that, for any $\left.\eta \in\right] \frac{\gamma}{1+\gamma}, 1[$, there holds $\left\|z_{k}-z^{\star}\right\|=O\left(\eta^{k}\right)$.

4.2. Sudoku and $s$-queens puzzles. In this part, we specialize the above result to Sudoku and $s$-queens puzzles. The examples of these two puzzles are provided in Figure 3 below.

\begin{tabular}{|l|l|l|l|l|l|l|l|l|}
\hline & 4 & & & & & & 9 & \\
\hline & & 8 & 3 & & 1 & 2 & & \\
\hline & & 5 & 8 & & 9 & 6 & & \\
\hline 6 & & & 2 & & 4 & & & 8 \\
\hline & 5 & 1 & & & & 4 & 2 & \\
\hline 2 & & & 6 & & 7 & & & 9 \\
\hline & & 6 & 7 & & 3 & 5 & & \\
\hline & & 2 & 1 & & 5 & 3 & & \\
\hline & 1 & & & & & & 7 & \\
\hline
\end{tabular}

(A) Sudoku puzzle

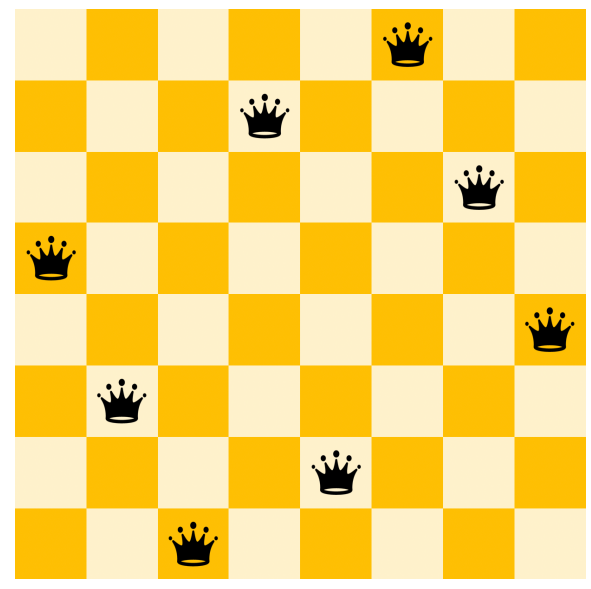

(B) Eight queens puzzle

FIgURE 3. Examples of Sudoku and eight queens. The goal of Sudoku is to complete the grid such that each row, column, and $3 \times 3$ square contains all the digits from 1 to 9 . The goal of eight queens is to place eight chess queens on an $8 \times 8$ board such that no two queens share the same row/column/diagonal.

4.2.1. Sudoku puzzle. A standard Sudoku puzzle is shown in Figure 3 (a), which we generalize to grids of size $s \times s$ with the basic setting and rules:

- a partially complete $s \times s$ grid is provided;

- each column, each row, and each of the s sub-grids of size $\sqrt{s} \times \sqrt{s}$ that compose the grid contain all of the digits from 1 to $s$. 
Based on the rules, we can easily formulate the Sudoku puzzle as a feasibility problem. Here we consider the formulation proposed in [28], which formulates Sudoku as a binary feasibility problem. We also refer to [24] for studies on the Sudoku puzzle and the Douglas-Rachford splitting method.

Each digit from 1 to $s$ is lifted to the set $[0,1]^{s}$, making the full puzzle an $s \times s \times s$ binary cube. Figure 4 (a) shows a feasible row of the lifted problem represented as a binary $s \times s$ square. Equivalently, we can say that any digit from 1 to $s$ is a permutation of unit vector $e=\{1,0, \ldots, 0\}$. This leads to four Sudoku feasibility constraints:

- each row of the cube, i.e. $C_{1}(:, j, k), j, k \in\{1, \ldots, s\}$, is the permutation of $e$; See Figure 4 (b);

- each column of the cube, i.e. $C_{2}(i,:, k), i, k \in\{1, \ldots, s\}$, is the permutation of $e$; See Figure 4 (c);

- each pillar of the cube, i.e. $C_{3}(i, j,:), i, j \in\{1, \ldots, s\}$, is the permutation of $e$; See Figure $4(\mathrm{~d})$;

- for each $k \in\{1, \ldots, s\}$, each of the $s$ sub-grids is the permutation of e, i.e. $C_{4}(\sqrt{s}(i-$ 1) $+1: \sqrt{s} i, \sqrt{s}(j-1)+1: \sqrt{s} j, k), i, j \in\{1, \ldots, \sqrt{s}\}$; See Figure 4 (e).

The partially completed grid forms the last constraint set

- $C_{5}$ is the constraint of the provided numbers.

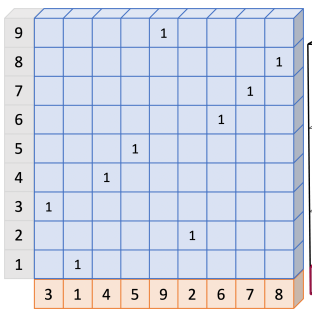

(A)

Lifted

row

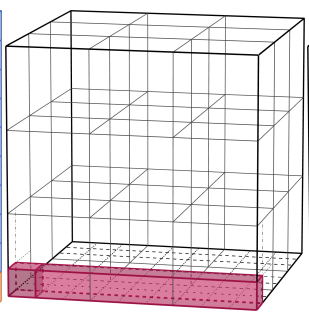

(B) $C_{1}$

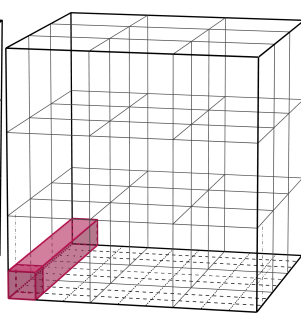

(C) $C_{2}$

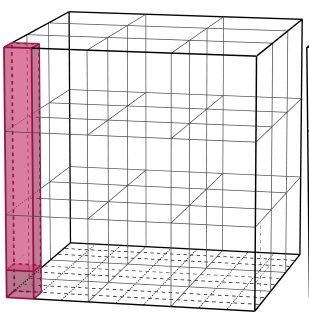

(D) $C_{3}$

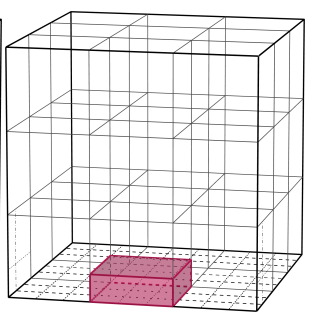

(E) $C_{4}$

FIGURE 4. Lifted Sudoku problem. (a) the lifted representation of a row of numbers. (b)-(e) show what is a lifted row/column/pillar/sub-grid respectively.

At this point, solving the Sudoku puzzle is equivalent to solve the following feasibility problem of the five constraint sets

$$
\text { find } \quad x \in \mathbb{R}^{s \times s \times s} \text { s.t. } x \in C_{1} \cap C_{2} \cap C_{3} \cap C_{4} \cap C_{5} .
$$

To obtain the product space formulation, let $\mathcal{H}=\underbrace{\mathbb{R}^{s \times s \times s} \times \cdots \times \mathbb{R}^{s \times s \times s}}_{5 \text { times }}, \mathcal{C} \stackrel{\text { def }}{=} C_{1} \times \cdots \times C_{5}$, and $\mathcal{S}=\left\{\boldsymbol{x}=\left(x_{i}\right)_{i} \in \mathcal{H}: x_{1}=\cdots=x_{5}\right\}$.

Proposition 4.1 (Local convergence of DR). For Sudoku puzzle (4.6) and Douglas-Rachford splitting (3.6), suppose that Assumptions (A.1)-(A.4) hold. Then $\left\{\boldsymbol{u}_{k}, x_{k}, z_{k}\right\}_{k \in \mathbb{N}}$ converges to $\left(\mathcal{K}\left(x^{\star}\right), x^{\star}, z^{\star}\right)$ with $z^{\star} \in \operatorname{Fix}\left(\mathscr{F}_{\mathrm{DR}}\right)$ being a fixed point and $x^{\star}=\frac{1}{5} \sum_{i=1}^{5} z_{i}^{\star}$. If, moreover, for $i=1, \ldots, 4, C_{i}$ is prox-regular at $x^{\star}$ for $x^{\star}-z_{i}^{\star}$, and the following non-degeneracy condition holds

$$
x^{\star}-z_{i}^{\star} \in \operatorname{int}\left(\mathscr{N}_{C_{i}}\left(x^{\star}\right)\right),
$$


then, for all $k$ large enough, there holds

- $u_{i, k}=x^{\star}$ for $i=1, \ldots, 4$,

- $\left\|z_{k}-z^{\star}\right\|=O\left(\eta^{k}\right)$ with $\eta=\frac{\sqrt{5}}{5}$.

Proof. Denote $\boldsymbol{x}_{k+1}=\mathcal{K}\left(x_{k+1}\right)$. From the updates of $\boldsymbol{x}_{k}$, we have that

$$
\boldsymbol{x}_{k+1}-\boldsymbol{x}^{\star}=\mathscr{P}_{\mathcal{S}}\left(z_{k}-z^{\star}\right)
$$

with $\mathscr{P}_{\mathcal{S}}=\frac{1}{5} \mathbf{1}_{5 \times 5} \otimes \operatorname{Id}_{s^{3} \times s^{3}}$, where $\mathbf{1}_{5 \times 5}$ stands for matrix of all 1 and $\otimes$ for Kronecker product. The separability of $\mathscr{P}_{\mathcal{C}}$ and the definition of projection operator lead to, for each $i=1, \ldots, 5$,

$$
\begin{aligned}
u_{i, k+1} & =\mathscr{P}_{C_{i}}\left(2 x_{k+1}-z_{i, k}\right) \\
\text { and } \quad u_{i, k+1}-u_{i}^{\star} & =\mathscr{P}_{C_{i}}\left(2 x_{k+1}-z_{i, k}\right)-\mathscr{P}_{C_{i}}\left(2 x^{\star}-z_{i}^{\star}\right) .
\end{aligned}
$$

Under non-degeneracy condition (4.7), apply the argument of Theorem 4.1 to obtain the finite convergence of $u_{i, k}$ for $i=1, \ldots, 4$. For $C_{5}$, since its projection operator is linear, we have

$$
\begin{aligned}
u_{5, k+1}-u_{5}^{\star} & =\mathscr{P}_{C_{5}}\left(2 x_{k+1}-z_{5, k}\right)-\mathscr{P}_{C_{5}}\left(2 x^{\star}-z_{5}^{\star}\right) \\
& =2 \mathscr{P}_{C_{5}}\left(x_{k+1}-x^{\star}\right)-\mathscr{P}_{C_{5}}\left(z_{5, k}-z_{5}^{\star}\right) .
\end{aligned}
$$

As a result, for $k$ large enough, there holds

$$
\boldsymbol{u}_{k+1}-\boldsymbol{u}^{\star}=2\left[\begin{array}{ll}
\mathbf{0}_{4 s^{3} \times 4 s^{3}} & \\
& \mathscr{P}_{C_{5}}
\end{array}\right] \mathscr{P}_{\mathcal{S}}\left(z_{k}-z^{\star}\right)-\left[\begin{array}{ll}
\mathbf{0}_{4 s^{3} \times 4 s^{3}} & \\
& \mathscr{P}_{C_{5}}
\end{array}\right]\left(z_{k}-z^{\star}\right) .
$$

Let $\mathscr{P}_{\mathcal{C}} \stackrel{\text { def }}{=}\left[\begin{array}{ll}\mathbf{0}_{4 s^{3} \times 4 s^{3}} & \\ & \mathscr{P}_{C_{5}}\end{array}\right]$ and back to $\boldsymbol{z}_{k+1}-\boldsymbol{z}^{\star}$, we obtain

$$
\begin{aligned}
\boldsymbol{z}_{k+1}-\boldsymbol{z}^{\star} & =\left(\boldsymbol{z}_{k}-\boldsymbol{z}^{\star}\right)+\left(\boldsymbol{u}_{k+1}-\boldsymbol{u}^{\star}\right)-\left(\boldsymbol{x}_{k+1}-\boldsymbol{x}^{\star}\right) \\
& =\left(\mathbf{I d}+2 \mathscr{P}_{\mathcal{C}} \mathscr{P}_{\mathcal{S}}-\mathscr{P}_{\mathcal{C}}-\mathscr{P}_{\mathcal{S}}\right)\left(\boldsymbol{z}_{k}-\boldsymbol{z}^{\star}\right),
\end{aligned}
$$

Since $\mathscr{P}_{C_{5}}$ is the projection operator onto a subspace, then $\mathscr{P}_{\mathcal{C}}$. As a result, the linear convergence rate is the cosine of the Friedrichs angle $\theta_{F}$ between the subspace of $\mathscr{P}_{\mathcal{C}}$ and that of $\mathscr{P}_{\mathcal{S}}$. We now need to analyze the singular values of $\mathscr{P}_{\mathcal{C}} \mathscr{P}_{\mathcal{S}}$, which essentially is the SVD of $\mathscr{P}_{C_{5}} \mathscr{P}_{S}$, where

$$
\mathscr{P}_{S}=\frac{1}{5} \mathbf{1}_{1 \times 5} \otimes \operatorname{Id}_{S^{3} \times s^{3}}
$$

We have

- $\mathscr{P}_{C_{5}}$ is diagonal matrix with only 0 and 1 ;

- $\mathscr{P}_{S}$ has a unique singular value which is $\frac{\sqrt{5}}{5}$.

As a result, $\mathscr{P}_{C_{5}} \mathscr{P}_{S}$ has only two singular values, which are 0 and $\frac{\sqrt{5}}{5}$. Hence we conclude the proof immediately.

Next, we present the result for damped Douglas-Rachford splitting (3.4).

Proposition 4.2 (Local convergence of dDR). For Sudoku puzzle (4.6) and damped DouglasRachford splitting (3.7), suppose that Assumptions (A.1)-(A.3) hold, and (3.7) is ran under the conditions of Theorem 3.1. Then $\left(\boldsymbol{u}_{k}, \boldsymbol{x}_{k}, \boldsymbol{z}_{k}\right)$ converges to $\left(\boldsymbol{x}^{\star}, \boldsymbol{x}^{\star}, \boldsymbol{z}^{\star}\right)$ with $\boldsymbol{z}^{\star}$ being a fixed point and $\boldsymbol{x}^{\star}$ a stationary point of $\min _{\boldsymbol{x}}\left\{\operatorname{dist}^{2}(\boldsymbol{x}, \mathcal{S})\right.$ s.t. $\left.\boldsymbol{x} \in \mathcal{C}\right\}$. If, moreover, non-degeneracy condition (4.7) holds for $C_{1, \ldots, 4}$, then, for all $k$ large enough, it holds

- $u_{i, k}=x^{\star}$ for $i=1, \ldots, 4$, 
- $\left\|z_{k}-z^{\star}\right\|=O\left(\eta^{k}\right)$ with $\eta=\frac{2 \gamma+5+\sqrt{25-16 \gamma^{2}}}{10(1+\gamma)}$.

Proof. From the updates of $x_{k+1}$, we have that

$$
\begin{aligned}
\boldsymbol{x}_{k+1}-\boldsymbol{x}^{\star} & =\frac{1}{1+\gamma}\left(\boldsymbol{z}_{k}+\boldsymbol{\gamma} \mathscr{P}_{\mathcal{S}}\left(\boldsymbol{z}_{k}\right)\right)-\frac{1}{1+\gamma}\left(\boldsymbol{z}^{\star}+\gamma \mathscr{P}_{b c S}\left(\boldsymbol{z}^{\star}\right)\right) \\
& =\frac{1}{1+\gamma}\left(\boldsymbol{z}_{k}-\boldsymbol{z}^{\star}\right)+\frac{\gamma}{1+\gamma} \mathscr{P}_{\mathcal{S}}\left(\boldsymbol{z}_{k}-\boldsymbol{z}^{\star}\right)
\end{aligned}
$$

with $\mathscr{P}_{\mathcal{S}}=\frac{1}{5} \mathbf{1}_{5 \times 5} \otimes \operatorname{Id}_{s^{3} \times s^{3}}$. For $\boldsymbol{u}_{k}$, the finite termination of $u_{i, k}, i=1, \ldots, 4$ follows from the proof of Proposition 4.1. For $C_{5}$, again we have

$$
u_{5, k+1}-u_{5}^{\star}=2 \mathscr{P}_{C_{5}}\left(x_{5, k+1}-x_{5}^{\star}\right)-\mathscr{P}_{C_{5}}\left(z_{5, k}-z_{5}^{\star}\right) .
$$

Let $\mathscr{P}_{\mathcal{C}} \stackrel{\text { def }}{=}\left[\begin{array}{ll}\mathbf{0}_{4 s^{3} \times 4 s^{3}} & \\ & \mathscr{P}_{C_{5}}\end{array}\right]$. It follows that

$$
\begin{aligned}
\boldsymbol{u}_{k+1}-\boldsymbol{u}^{\star} & =2 \mathscr{P}_{\mathcal{C}}\left(\boldsymbol{x}_{k+1}-\boldsymbol{x}^{\star}\right)-\mathscr{P}_{\mathcal{C}}\left(z_{k}-z^{\star}\right) \\
& =\frac{2}{1+\gamma} \mathscr{P}_{\mathcal{C}}\left(z_{k}-z^{\star}\right)+\frac{2 \gamma}{1+\gamma} \mathscr{P}_{\mathcal{C}} \mathscr{P}_{\mathcal{S}}\left(z_{k}-z^{\star}\right)-\mathscr{P}_{\mathcal{C}}\left(z_{k}-z^{\star}\right) \\
& =\frac{1-\gamma}{1+\gamma} \mathscr{P}_{\mathcal{C}}\left(z_{k}-z^{\star}\right)+\frac{2 \gamma}{1+\gamma} \mathscr{P}_{\mathcal{C}} \mathscr{P}_{\mathcal{S}}\left(z_{k}-z^{\star}\right) .
\end{aligned}
$$

Back to $z_{k+1}-z^{\star}$, we see that

$$
\begin{aligned}
\boldsymbol{z}_{k+1}-\boldsymbol{z}^{\star}= & \left(\boldsymbol{z}_{k}-\boldsymbol{z}^{\star}\right)+\left(\boldsymbol{u}_{k+1}-\boldsymbol{u}^{\star}\right)-\left(\boldsymbol{x}_{k+1}-\boldsymbol{x}^{\star}\right) \\
= & \left(\boldsymbol{z}_{k}-\boldsymbol{z}^{\star}\right)+\frac{1-\gamma}{1+\gamma} \mathscr{P}_{\mathcal{C}}\left(\boldsymbol{z}_{k}-\boldsymbol{z}^{\star}\right)+\frac{2 \gamma}{1+\gamma} \mathscr{P}_{\mathcal{C}} \mathscr{P}_{\mathcal{S}}\left(\boldsymbol{z}_{k}-\boldsymbol{z}^{\star}\right) \\
& \quad-\frac{1}{1+\gamma}\left(z_{k}-z^{\star}\right)-\frac{\gamma}{1+\gamma} \mathscr{P}_{\mathcal{S}}\left(z_{k}-\boldsymbol{z}^{\star}\right) \\
= & \frac{1}{1+\gamma}\left(\boldsymbol{\gamma} \mathbf{I d}+2 \gamma \mathscr{P}_{\mathcal{C}} \mathscr{P}_{\mathcal{S}}+(1-\gamma) \mathscr{P}_{\mathcal{C}}-\gamma \mathscr{P}_{\mathcal{S}}\right)\left(z_{k}-z^{\star}\right) \\
= & \frac{1}{1+\gamma}\left(\gamma\left(\mathbf{I d}+2 \mathscr{P}_{\mathcal{C}} \mathscr{P}_{\mathcal{S}}-\mathscr{P}_{\mathcal{C}}-\mathscr{P}_{\mathcal{S}}\right)+\mathscr{P}_{\mathcal{C}}\right)\left(z_{k}-z^{\star}\right) .
\end{aligned}
$$

Denote $M_{\gamma}=\frac{1}{1+\gamma}\left(\gamma\left(\mathbf{I d}+2 \mathscr{P}_{\mathcal{C}} \mathscr{P}_{\mathcal{S}}-\mathscr{P}_{\mathcal{C}}-\mathscr{P}_{\mathcal{S}}\right)+\mathscr{P}_{\mathcal{C}}\right)$. Let $p, q$ be the rank of $\mathscr{P}_{\mathcal{C}}$ and $\mathscr{P}_{\mathcal{S}}$, respectively, and assume $p \leq q$ (for the case $p \geq q$, similar result can be obtained). Based on [22], there exists an orthogonal matrix $U$ such that

$\mathscr{P}_{\mathcal{C}}=U\left[\begin{array}{cc|cc}\operatorname{Id}_{p} & 0 & 0 & 0 \\ 0 & 0_{p} & 0 & 0 \\ \hline 0 & 0 & 0_{q-p} & 0 \\ 0 & 0 & 0 & 0_{n-p-q}\end{array}\right] U^{*} \quad$ and $\quad \mathscr{P}_{\mathcal{S}}=U\left[\begin{array}{cc|cc}\alpha^{2} & \alpha \beta & 0 & 0 \\ \alpha \beta & \beta^{2} & 0 & 0 \\ \hline 0 & 0 & \operatorname{Id}_{q-p} & 0 \\ 0 & 0 & 0 & 0_{n-p-q}\end{array}\right] U^{*}$,

where $\alpha=\operatorname{diag}\left(\cos \left(\theta_{1}\right), \ldots, \cos \left(\theta_{p}\right)\right)$ and $\beta=\operatorname{diag}\left(\sin \left(\theta_{1}\right), \ldots, \sin \left(\theta_{p}\right)\right)$ with $\theta_{i, i=1, . . p}$ being the principal angles between the subspaces of $\mathscr{P}_{\mathcal{C}}$ and $\mathscr{P}_{\mathcal{S}}$. Consequently,

$$
\mathbf{I d}+2 \mathscr{P}_{\mathcal{C}} \mathscr{P}_{\mathcal{S}}-\mathscr{P}_{\mathcal{C}}-\mathscr{P}_{\mathcal{S}}=U\left[\begin{array}{cc|cc}
\alpha^{2} & \alpha \beta & 0 & 0 \\
-\alpha \beta & \alpha^{2} & 0 & 0 \\
\hline 0 & 0 & 0_{q-p} & 0 \\
0 & 0 & 0 & \operatorname{Id}_{n-p-q}
\end{array}\right] U^{*}
$$


Therefore, we have

$$
M_{\gamma}=\frac{1}{1+\gamma} U\left[\begin{array}{cc|cc}
\gamma \alpha^{2}+\operatorname{Id}_{p} & \gamma \alpha \beta & 0 & 0 \\
-\gamma \alpha \beta & \gamma \alpha^{2} & 0 & 0 \\
\hline 0 & 0 & 0_{q-p} & 0 \\
0 & 0 & 0 & \gamma \operatorname{Id}_{n-p-q}
\end{array}\right] U^{*}
$$

Clearly, 0 and $\frac{\gamma}{1+\gamma}$ are two eigenvalues of the matrix. For the top left block of the above matrix, as it is block diagonal, we have the following characteristic polynomial

$$
0=\prod_{i=1}^{p}\left(\left(\frac{\gamma \alpha_{i}^{2}+1}{1+\gamma}-\lambda\right)\left(\frac{\gamma \alpha_{i}^{2}}{1+\gamma}-\lambda\right)+\frac{\gamma^{2} \alpha_{i}^{2} \beta_{i}^{2}}{(1+\gamma)^{2}}\right) .
$$

Solving the quadratic equation for each $i$, we have

$$
\lambda_{i}=\frac{2 \gamma \alpha_{i}^{2}+1 \pm \sqrt{1-4 \gamma^{2} \alpha_{i}^{2} \beta_{i}^{2}}}{2(1+\gamma)}
$$

As in the proof of Proposition 4.1, we have that $\alpha_{i}=\frac{\sqrt{5}}{5}$ for all $i=1, \ldots, p$. Therefore, $M_{\gamma}$ has only 4 distinct eigenvalues, which are

$$
0, \quad \frac{2 \gamma+5-\sqrt{25-16 \gamma^{2}}}{10(1+\gamma)}, \quad \frac{\gamma}{1+\gamma} \text { and } \quad \frac{2 \gamma+5+\sqrt{25-16 \gamma^{2}}}{10(1+\gamma)}
$$

We also have

$$
\begin{aligned}
& \left.\left.\frac{\gamma}{1+\gamma} \leq \frac{2 \gamma+5+\sqrt{25-16 \gamma^{2}}}{10(1+\gamma)}: \gamma \in\right] 0,1\right] \\
& \left.\frac{\gamma}{1+\gamma} \geq\left|\frac{2 \gamma+5+\sqrt{25-16 \gamma^{2}}}{10(1+\gamma)}\right|: \gamma \in\right] 1,+\infty[
\end{aligned}
$$

Next, we focus on $\gamma \in] 0,1]$, and show that $\eta=\frac{2 \gamma+5+\sqrt{25-16 \gamma^{2}}}{10(1+\gamma)}$ is the convergence rate. To this end, we need to show that $\eta$ is semi-simple. Let $M_{p}=\left[\begin{array}{cc}\gamma \alpha^{2}+\operatorname{Id}_{p} & \gamma \alpha \beta \\ -\gamma \alpha \beta & \gamma \alpha^{2}\end{array}\right]$. Since $\alpha=\frac{\sqrt{5}}{5}$ is a $p$ 'th order root, we can simplify $M_{p}$ as:

$$
M_{p}=\frac{1}{5}\left[\begin{array}{cc}
(\gamma+5) \operatorname{Id}_{p} & 2 \gamma \operatorname{Id}_{p} \\
-2 \gamma \operatorname{Id}_{p} & \gamma \operatorname{Id}_{p}
\end{array}\right]
$$

As a result, we have

$$
\begin{aligned}
\operatorname{rank}\left(M_{p}-\eta \operatorname{Id}_{2 p}\right) & =\operatorname{rank}\left(\left[\begin{array}{cc}
(\gamma+5-5 \eta) \operatorname{Id}_{p} & 2 \gamma \operatorname{Id}_{p} \\
-2 \gamma \operatorname{Id}_{p} & (\gamma-5 \eta) \operatorname{Id}_{p}
\end{array}\right]\right)=p, \quad \text { and } \\
\operatorname{rank}\left(\left(M_{p}-\eta \operatorname{Id}_{2 p}\right)^{2}\right) & =\operatorname{rank}\left(\left[\begin{array}{cc}
\left.(\gamma+5-5 \eta)^{2}-4 \gamma^{2}\right) \operatorname{Id}_{p} & 2 \gamma(\gamma+5-10 \eta) \operatorname{Id}_{p} \\
-2 \gamma(\gamma+5-10 \eta) \operatorname{Id}_{p} & \left((\gamma-5 \eta)^{2}-4 \gamma^{2}\right) \operatorname{Id}_{p}
\end{array}\right]\right)=p,
\end{aligned}
$$

which means that $\eta$ is semi-simple by Definition 2.6, and then we conclude the linear rate of convergence.

Remark 4.5. The proof of the two propositions above is dimension independent, which means the results hold true for all puzzle sizes of perfect squares $s$ with $s \geq 4$; see Section 5 for numerical illustrations. 
4.3. $s$-queens puzzle. The rule of eight queens puzzle is rather simple: placing eight chess queens on an $8 \times 8$ chessboard so that no two queens threaten each other. The size of the puzzle can be generalized to any size $s \times s$ with $s \geq 4$, while there is no solution for $s=2,3$ and a trivial solution for $s=1$ which is obvious.

We follow the setting of [28]. On the chessboard, as there are four directions (horizontal, vertical and two diagonal directions) for the queen to move, we have four constraint sets for the problem:

- $C_{1}$ : each row has only one queen;

- $C_{2}$ : each column has only one queen;

- $C_{3}$ : each diagonal direction southeast-northwest, there is at most one queen;

- $C_{4}$ : each diagonal direction southwest-northeast, there is at most one queen.

Now we can formulate the $s$-queens puzzle as a feasibility problem of four sets

$$
\text { find } \quad x \in \mathbb{R}^{s \times s} \text { s.t. } x \in C_{1} \cap C_{2} \cap C_{3} \cap C_{4} \text {. }
$$

Since all the sets above are binary, so is the set $\mathcal{C} \stackrel{\text { def }}{=} C_{1} \times \cdots \times C_{4}$, as a result finite convergence can be obtained under the conditions of Theorems 4.1 and 4.2, for the standard DouglasRachford and the damped one, respectively.

\section{NUMERICAL RESULTS}

We now provide numerical results on Sudoku and $s$-queens puzzles to support our theoretical findings. Before analyzing the convergence rates, we first compare the performance of standard Douglas-Rachford splitting method (3.2) and damped one (3.4) on how successful are they when applied to solve these two puzzles. i.e. how often each method finds a feasible point.

The comparison is shown in Table 1. For (3.4), two choices of $\gamma$ are considered: $\gamma=\frac{1}{5} \in$ ],$\sqrt{3 / 2}-1[$ suggested by Lemma 3.1 and $\gamma=99$ with online tracking rule suggested in [12, Remark 4]. For both methods, the iteration is terminated if either a stopping criterion is met or $10^{4}$ steps of iteration are reached, then we verify the output. Also, a minimal 100 number of iteration is set. For a given puzzle, each method is repeated $10^{3}$ times with different initialization for each running.

For Sudoku, the size of both puzzles are $9 \times 9$ : "Puzzle 1" is provided with 37 digits, hence is easy; "Puzzle 2" has 22 given digits and is more difficult than "Puzzle 1"

- the standard DR solves both puzzles with $100 \%$ success rate, while the dDR with $\gamma=\frac{1}{5}$ fails all tests. The dDR with $\gamma=99$ succeeds on "Puzzle 1", and the rate drops to about $88 \%$ for "Puzzle 2";

- in terms of number of iterations, the sDR needs much less number of iterations compared to those of the dDR with $\gamma=99$.

For the $s$-queens puzzle, two different sizes are considered: $s=8$ for "Puzzle 1 " and $s=16$ for "Puzzle 2".

https://en.wikipedia.org/wiki/Eight_queens_puzzle

For $9 \times 9$ Sudoku, to ensure the uniqueness of solutions, the smallest number of given digits of the puzzle is 17. See https://www.technologyreview.com/2012/01/06/188520/ mathematicians-solve-minimum-sudoku-problem/ 
- similar to Sudoku, the dDR with $\gamma=\frac{1}{5}$ fails all tests. This time, between the sDR and the dDR with $\gamma=99$, neither achieves $100 \%$ success rate with the dDR being better than sDR.

- in terms of number of iteration, same as the Sudoku case, the sDR is better.

The above observation, in particular, the failure of dDR with $\gamma=\frac{1}{5}$, is in contrast to Example 3.1. One possible reason leads to the failure of the dDR with $\gamma=\frac{1}{5}$ is that set $C$ is finite and the dDR can not escape bad local stationary point with small value of $\gamma$.

TABLE 1. Comparison of success rate of standard DR and damped DR for solving Sudoku and $s$-queens puzzles over 1,000 random initializations.

\begin{tabular}{|c|c|c|c|c|c|c|c|}
\hline \multicolumn{2}{|c|}{} & \multicolumn{3}{c|}{ Puzzle 1} & \multicolumn{3}{c|}{ Puzzle 2 } \\
\cline { 3 - 8 } \multicolumn{2}{c|}{} & sDR & dDR $\gamma=\frac{1}{5}$ & dDR $\gamma=99$ & sDR & dDR $\gamma=\frac{1}{5}$ & dDR $\gamma=99$ \\
\hline \multirow{3}{*}{ Sudoku } & success rate & $100 \%$ & 0 & $100 \%$ & $100 \%$ & 0 & $89.7 \%$ \\
\cline { 2 - 8 } & avg. \# of itr. & 114 & 184 & 2710 & 408 & 184 & 5409 \\
\hline \multirow{2}{*}{$s$-queens } & success rate & $94.8 \%$ & 0 & $98.0 \%$ & $90.2 \%$ & 0 & $92.2 \%$ \\
\cline { 2 - 8 } & avg. \# of itr. & 653 & 100 & 2812 & 1286 & 100 & 3618 \\
\hline
\end{tabular}

5.1. Sudoku puzzle. We consider three different puzzle sizes for Sudoku to verify out results: 4, 9, and 16, which are shown in Figure 5 (a)-(c). In each size, we have 4, 32, and 128 coefficients provided respectively. The convergence behavior of the standard Douglas-Rachford splitting method can be seen in the second and third rows of Figure 5, from which we observe that for all puzzles,

- finite termination of $u_{i, k}, i=1, \ldots, 4$ : in the second row of Figure 5, we provide the $\ell_{0}$ pseudo-norms of $\left\|u_{i, k}-u_{i}^{\star}\right\|_{0}, i=1, \ldots, 4$ to show the mismatch between $u_{i, k}$ and $u_{i}^{\star}$. We observed that, for each $i \in\{1,2,3,4\},\left\|u_{i, k}-u_{i}^{\star}\right\|_{0}$ reaches 0 in finite steps, which means the finite termination.

- local linear convergence In the last row of Figure 5, we provide the convergence behaviors of $\left\|\boldsymbol{u}_{k}-\boldsymbol{u}^{\star}\right\|$ (which actually reduces to $\left\|u_{5, k}-u_{5}^{\star}\right\|$ ), $\left\|x_{k}-x^{\star}\right\|$ and $\left\|z_{k}-z^{\star}\right\|$. Taking $\left\|\boldsymbol{z}_{k}-\boldsymbol{z}^{\star}\right\|$ for example, we have that its convergence has two different regimes: sub-linear rate from the beginning, and linear rate locally. The magenta dashed line is our theoretical estimation of the linear convergence rate and the slope of the line is $\frac{\sqrt{5}}{5}$. For all three different puzzle sizes, the local linear convergence rate is $\frac{\sqrt{5}}{5} \approx 0.45$, which confirms that the rate is independent of puzzle size.

5.2. $s$-queens puzzle. For the $s$-queens puzzle, we also consider three different puzzle sizes: $s=8,16$, and 25, which are shown in Figure 6 (a)-(c). The convergence behaviors of the Douglas-achford splitting method are shown in the second row of Figure 6. Since all the constraint sets are binary, we observe finite convergence for the algorithm, which complies with our theoretical results.

5.3. The damped Douglas-Rachford splitting. We conclude our numerical experiments by showing the local linear convergence the damped Douglas-Rachford splitting method with $\gamma=$ 99. The results on the Sudoku puzzle of size $9 \times 9$ and eight queens puzzle of size $8 \times 8$ are 


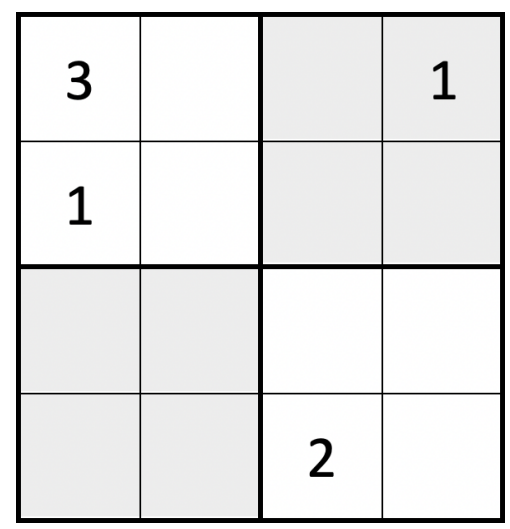

(A) Size $4 \times 4$

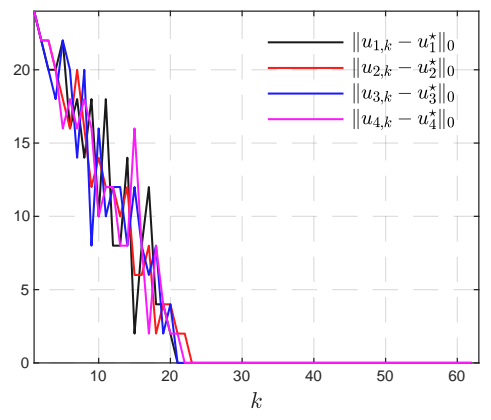

(D) Size 4: convergence of $u_{i, k}$

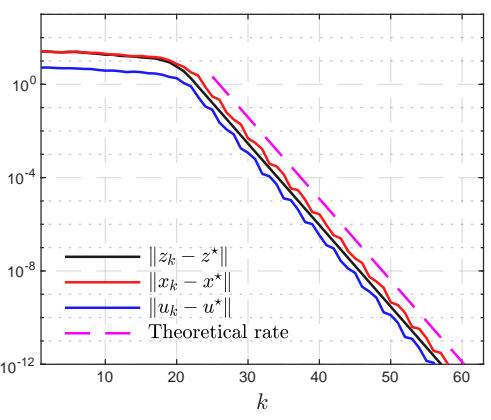

(G) Size 4: convergence of $\left(\boldsymbol{u}_{k}, x_{k}, z_{k}\right)$

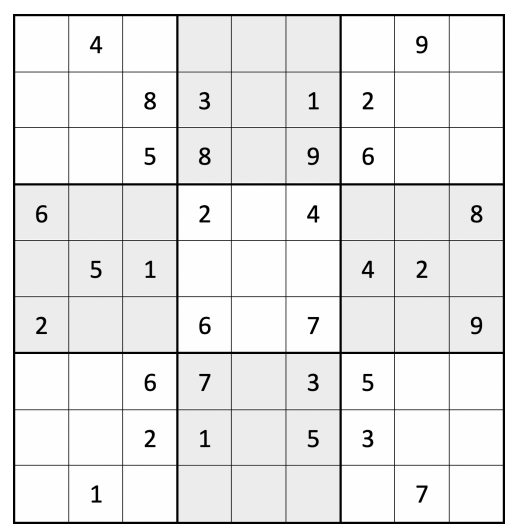

(B) Size $9 \times 9$

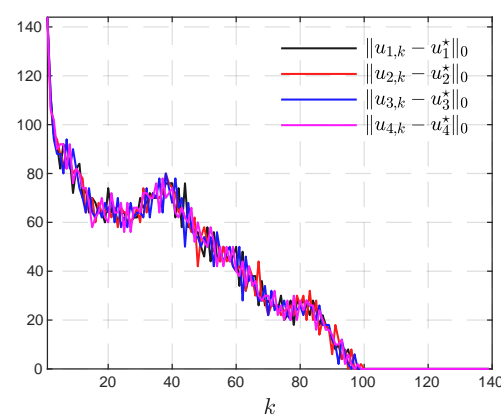

(E) Size 9: convergence of $u_{i, k}$

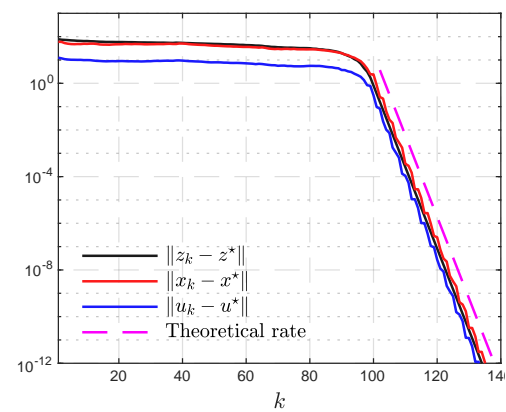

(H) Size 9: convergence of $\left(\boldsymbol{u}_{k}, x_{k}, z_{k}\right)$

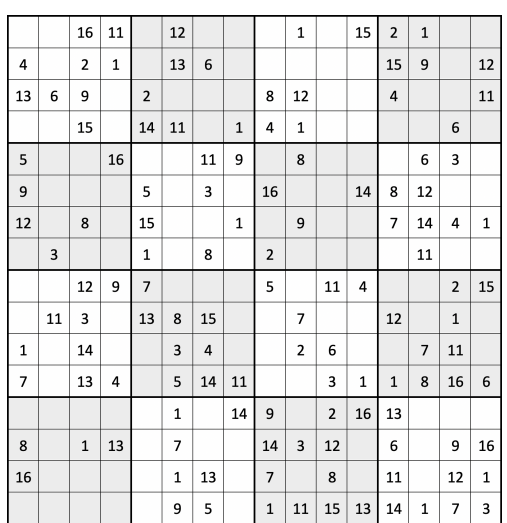

(C) Size $16 \times 16$

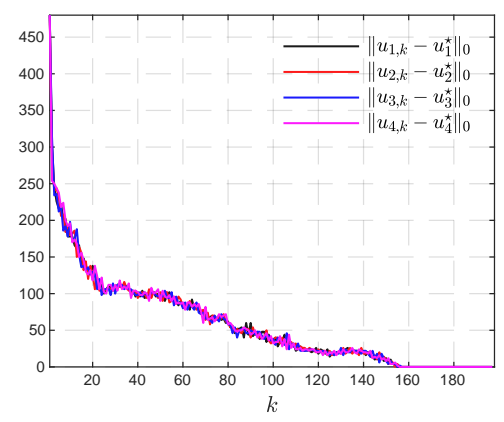

(F) Size 16: convergence of $u_{i, k}$

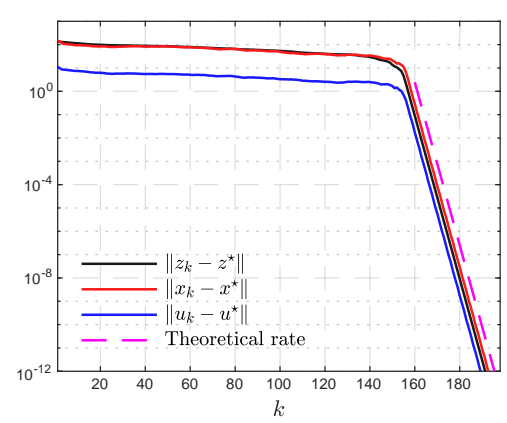

(I) Size 16: convergence of $\left(\boldsymbol{u}_{k}, x_{k}, z_{k}\right)$

FIGURE 5. Different sizes of Sudoku puzzles and convergence observations.

shown below in Figure 7. For both plots, the magenta line is our theoretical estimation of the local linear rate:

- for Sudoku puzzle, the slope of the magenta line is $\frac{2 \gamma+5+\sqrt{25-16 \gamma^{2}}}{10(1+\gamma)} \approx 0.86$.

- for eight queens puzzle, the slope of the magenta line is $\frac{\gamma}{1+\gamma} \approx 0.17$.

Again, our theoretical estimations are tight. We omit the plots of the dDR with $\gamma=99$ as they are very similar to those of Figure 7, except the different rates of local linear convergence. 


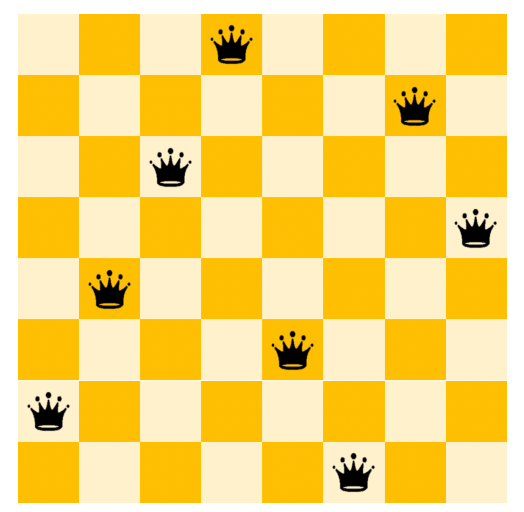

(A) Size 8

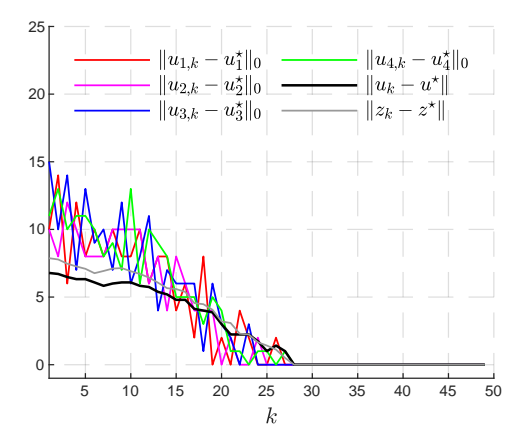

(D) Size 8

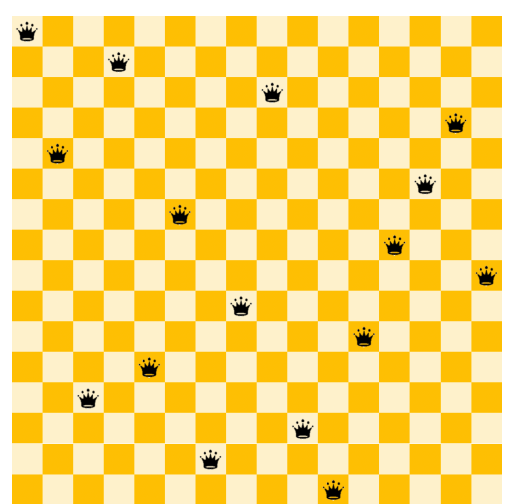

(B) Size 16

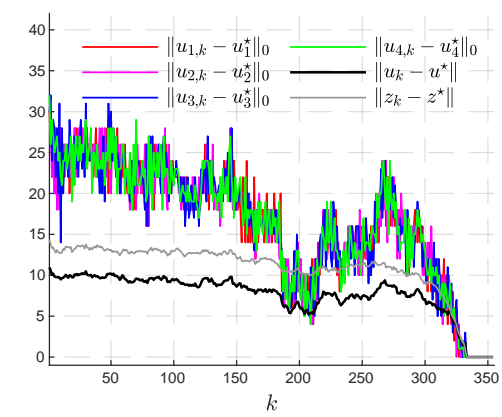

(E) Size 16

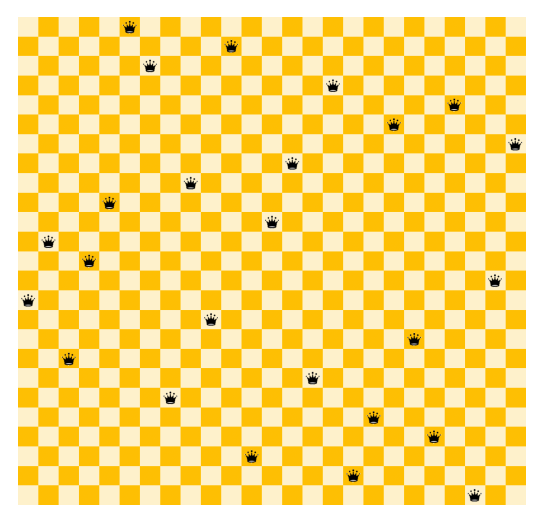

(C) Size 25

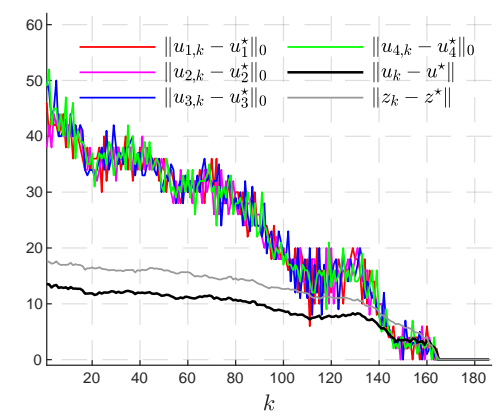

(F) Size 25

FIGURE 6. Different sizes of queens puzzles and convergence observations.

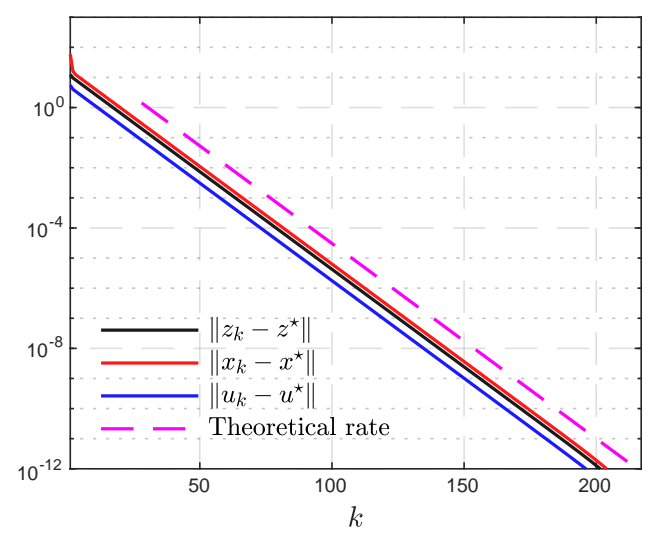

(A) Sudoku puzzle

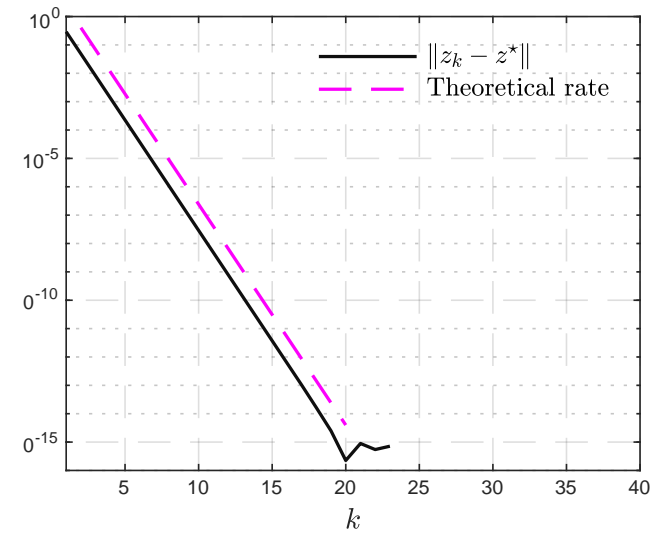

(B) Eight queens puzzle

FIGURE 7. Local linear convergence of the damped Douglas-Rachford for Sudoku puzzle and eight queens puzzle. Note again that convergence does not imply finding a solution to the feasibility problem.

\section{Conclusions}

In this paper, we studied local convergence properties of the Douglas-Rachford splitting method when applied to solve non-convex feasibility problems. Under a proper non-degeneracy 
condition, both finite convergence and local linear convergence were proved for the standard Douglas-Rachford splitting and a damped version of the method. Understanding when the methods fail, especially for the damped Douglas-Rachford splitting, require further study on the property of the methods.

\section{Acknowledgments}

We would like to thank Guoyin Li for helpful discussions on the convergence of the DouglasRachford splitting for non-convex optimization. Most of the work was done while the authors were at University of Cambridge. The second author was partly supported by Leverhulme trust, Newton trust and the EPSRC centre "EP/N014588/1". The first author acknowledges the funding from EPSRC Grant No. "EP/L016516/1" for the Cambridge Centre for Analysis. Both the authors were supported by the Cantab Capital Institute for Mathematics of Information.

\section{REFERENCES}

[1] J. von Neumann, Functional operators, vol. 2 (Annals of Mathematics Studies, no. 22), Princeton, NJ, 1950.

[2] L.M. Bregman, The method of successive projection for finding a common point of convex sets, Sov. Math. Dok. 162 (1965), 688-692.

[3] A. Cegielski, A. Suchocka, Relaxed alternating projection methods, SIAM J. Optim. 19 (2008), 1093-1106.

[4] D.R. Luke, Relaxed averaged alternating reflections for diffraction imaging, Inverse Probl. 21 (2004), 37.

[5] P.L. Lions, B. Mercier, Splitting algorithms for the sum of two nonlinear operators, SIAM J. Nume. Anal. 16 (1979), 964-979.

[6] P.L. Combettes, J.C. Pesquet, Proximal splitting methods in signal processing. In Fixed-Point Algorithms for Inverse Problems in Science and Engineering, pp. 185-212, Springer, 2011.

[7] D.W. Peaceman, H.H. Rachford, Jr., The numerical solution of parabolic and elliptic differential equations, J. Soc. Indust. Appl. Math. 3 (1955), 28-41.

[8] J. Douglas, H.H. Rachford, On the numerical solution of heat conduction problems in two and three space variables, Trans. Amer. Math. Soc. 82 (1956), 421-439.

[9] H.H. Bauschke, M.N. Dao, On the finite convergence of the douglas-rachford algorithm for solving (not necessarily convex) feasibility problems in euclidean spaces, SIAM J. Optim. 27 (2017), 507-537.

[10] H. Attouch, J. Bolte, P. Redont, A. Soubeyran, Proximal alternating minimization and projection methods for nonconvex problems: An approach based on the Kurdyka-Łojasiewicz inequality, Math. Oper. Res. 35 (2010), 438-457.

[11] F.J.A. Artacho, J.M. Borwein, Global convergence of a non-convex douglas-rachford iteration, J. Global Optim. 57 (2013), 753-769.

[12] G. Li, T.K. Pong, Douglas-rachford splitting for nonconvex optimization with application to nonconvex feasibility problems, Math. Program. 159 (2016), 371-401.

[13] H.H. Bauschke, M.N. Dao, D. Noll, H.M. Phan, On slater's condition and finite convergence of the douglas-rachford algorithm for solving convex feasibility problems in euclidean spaces, J. Global Optim. 65 (2016), 329-349.

[14] J. Liang, J. Fadili, G. Peyré, Local convergence properties of Douglas-Rachford and alternating direction method of multipliers, J. Optim. Theory Appl. 172 (2017), 874-913.

[15] A.S. Lewis, Active sets, nonsmoothness, and sensitivity, SIAM J. Optim. 13 (2003), 702-725.

[16] S.-Y. Matsushita, L. Xu, On the finite termination of the douglas-rachford method for the convex feasibility problem, Optimization, 65 (2016), 2037-2047.

[17] H.M. Phan, Linear convergence of the douglas-rachford method for two closed sets, Optimization, 65 (2016), 369-385.

[18] R. Hesse, D.R. Luke, Nonconvex notions of regularity and convergence of fundamental algorithms for feasibility problems, SIAM J. Optim. 23 (2013), 2397-2419. 
[19] R. Hesse, D.R. Luke, P. Neumann, Projection methods for sparse affine feasibility: Results and counterexamples, Technical Report, 2013.

[20] H.H. Bauschke, J.M. Borwein, On projection algorithms for solving convex feasibility problems, SIAM Rev. 38 (1996), 367-426.

[21] C.D. Meyer, Matrix Analysis and Applied Linear Algebra, vol. 2. SIAM, 2000.

[22] H.H. Bauschke, J.Y. Bello Cruz, T.T.A. Nghia, H.M. Pha, X. Wang, Optimal rates of linear convergence of relaxed alternating projections and generalized douglas-rachford methods for two subspaces, Numer. Algo. 73 (2016), 33-76.

[23] R. Hesse, D. R. Luke, P. Neumann, Alternating projections and Douglas-Rachford for sparse affine feasibility, IEEE Trans. Signal Process. 62 (2014), 4868-4881.

[24] F.J.A. Artacho, J.M. Borwein, M.K. Tam, Recent results on douglas-rachford methods for combinatorial optimization problems, J. Optim. Theory Appl. 163 (2014), 1-30.

[25] H.H. Bauschke, D. Noll, On the local convergence of the douglas-rachford algorithm, Archiv der Math. 102 (2014), 589-600.

[26] F.J.A. Artacho, J.M. Borwein, M.K. Tam, Global behavior of the douglas-rachford method for a nonconvex feasibility problem, J. Global Optim. 65 (2016), 309-327.

[27] A. Themelis, P. Patrinos, Douglas-rachford splitting and admm for nonconvex optimization: Tight convergence results, SIAM J. Optim. 30 (2020), 149-181.

[28] J. Schaad, Modeling the 8-queens problem and sudoku using an algorithm based on projections onto nonconvex sets, PhD thesis, University of British Columbia, 2010. 